\title{
Models of covariation-based causal judgment: A review and synthesis
}

\author{
José C. Perales \\ University of Granada, Granada, Spain \\ AND \\ DaVID R. SHANKS \\ University College, London, England
}

\begin{abstract}
Causal judgment is assumed to play a central role in prediction, control, and explanation. Here, we consider the function or functions that map contingency information concerning the relationship between a single cue and a single outcome onto causal judgments. We evaluate normative accounts of causal induction and report the findings of an extensive meta-analysis in which we used a cross-validation model-fitting method and carried out a qualitative analysis of experimental trends in order to compare a number of alternative models. The best model to emerge from this competition is one in which judgments are based on the difference between the amount of confirming and disconfirming evidence. A rational justification for the use of this model is proposed.
\end{abstract}

Over the past 25 or so years, many researchers have sought to characterize the mechanisms by which people form causal beliefs from covariation information. This can be seen as a special case of the fundamental problem of associative learning (in a broad sense)—namely, the problem of describing how learned beliefs relate to the environmental contingencies on which they are based. Causal beliefs are influenced by a range of factors such as the temporal (Shanks, Pearson, \& Dickinson, 1989; see also Buehner \& May, 2003) and spatial (Glautier, 2002) distribution of events, as well as by more top-down factors such as causal expectations (Waldmann \& Hagmayer, 2001). Yet within the total space defined by the attributes that influence causal learning, research has tended to occupy a rather restricted region: Specifically, numerous studies have held constant all other factors (e.g., spatial and temporal relationships) and varied only covariation information - that is, information about the frequencies of conjunctions and disjunctions between a target cause and effect. This research has thus been focused on a seemingly rather straightforward problem: to characterize the function that maps covariation information onto causal beliefs. Until an adequate description of this function emerges, we cannot be said to have even the beginnings of an understanding of causal knowledge or, since causal knowledge is central to cognition (Sloman, 2005), of cognition itself.

This seemingly simple quest has proven to be a formidable challenge. However, there is no lack of candidate theories. The problem, rather, is that the core components of the theories are surrounded, in Kuhnian fashion, by a penumbra of additional assumptions that can often be manipulated to explain away awkward results. The end result is that we have not even been able to discriminate at the highest level of the theoretical hierarchy between associative and rule-based accounts, never mind to make finer discriminations between theories within each class. Moreover, research practice has exacerbated the problem. Researchers tend to design and run new experiments to distinguish between theories (often only between two theories), focusing on the parts of the experimental space where their favored theory is a priori likely to be most successful. There is little attempt to ensure that the favored theory adequately handles the historical record of prior experiments.

In the present article, we take a new approach in an attempt to circumvent, at least partially, these difficulties. We report the results of a meta-analysis of historical causal learning studies that have manipulated contingency information, and we employ a cross-validation methodology, suitable for comparing models that make incommensurable assumptions, to try to find the model that provides the best overall account of the data. Although any given theory can argue away the results of a particular study by changing some of its peripheral assumptions or by arguing that some aspect of the experimental procedure violated model assumptions, one can hardly take this line in the face of a decisive meta-analysis. The model that proved best is surprising in that it has not received the most attention in the literature.

Interevent causal induction has been studied in the laboratory by means of tasks in which a candidate cause 
and an effect appear in a more or less correlated manner (Smedslund, 1963; see Shanks, Holyoak, \& Medin, 1996, for a review). The learning of that relationship is later assessed by requesting a subjective estimate or a predictive response of some kind. In the most common version of this procedure, the task is divided into a series of discrete temporal intervals (trials), in each of which the two target events may or may not occur. The event that is presented first in each trial is commonly called the cue, and the one that is presented second, the outcome (typically, the cue is the candidate cause, and the outcome is the effect, but the causal order can be reversed, in which case the cue represents the effect and the outcome the candidate cause; see Perales, Catena, \& Maldonado, 2004; Waldmann, 2000; Waldmann \& Holyoak, 1992, 1997). Combining the presence/absence values of the cause and the effect yields four trial types. In $\boldsymbol{a}$ type trials, both the cause and the effect are present; in $\boldsymbol{b}$ type trials, the cause is present, but the effect is absent; in $c$ type trials, the cause is absent, but the effect is present; and finally, in $\boldsymbol{d}$ type trials, both the cause and the effect are absent.

A variety of models has been proposed to explain how people extract causal knowledge from covariation. Normbased models are inspired by rational analyses of causality (given a certain criterion for rationality; see Cheng, 1993) and assume that humans acquire causal knowledge by applying psychological operations that resemble rational strategies. In a weaker sense, norm-based models maintain that the output of psychological causal-induction processes conforms to the output of rational/normative induction independently of the operations that lead the reasoner to that output. All instances of nonnormative or algorithmic models, on the other hand, propose a mathematical algorithm, or a chain of such algorithms, to describe the psychological processes for drawing causal inductions. These models are nonnormative in the sense that they are not bounded to any particular norm of rationality, although it is implicitly assumed that they provide adaptive solutions for causal induction problems in real life.

Previewing the content of this article, we have collected current models of simple covariation-based causal induction of both types, under the restriction that they are still viable (although sometimes partial) accounts of the available data. Judgments predicted by these models are contrasted with actual judgments from a (nontrivial) selection of 114 experimental conditions from 19 experiments. The experiment selection criteria were carefully established to allow all of the models under consideration to be fairly assessed. These criteria impose strict constraints on the procedure followed in those experiments: In all of them, the covariation information is provided on a trial-by-trial basis, a standard causal probe wording is used to assess causal knowledge, and no more than one candidate cause and one effect are under assessment (see below).

\section{The Computational Goals of Covariation-Based Causal Induction}

Until very recently, most psychological models of covariation-based causal induction assumed that capturing a causal link was a goal that could be attained by the operation of a simple algorithm that translated covariational information into a value of subjective causal strength (between a potential cause and an effect), which could be later translated into a numerical or verbal judgment.

A more thorough analysis of the computational goals of causal inference convincingly demonstrates that, as a normative approach, this assumption is too simplistic (Danks, 2002; Glymour, 2001; Lagnado \& Sloman, 2004; Pearl, 2000; Sloman \& Lagnado, 2004; Tenenbaum \& Griffiths, 2001). Imagine that an individual has taken a pill on two of the last four days and has suffered from headaches on the same two days on which he or she took the pill but not on the other two days. In this case, the correlation between taking the pill and suffering headaches in that time window is maximal; nonetheless, most people would still conclude that more extensive evidence is needed to be sure that taking the pill caused the headaches. First, one would need to know about the presence or absence of other potential causes of the same effect, and second, more trials would be necessary for one to be certain that the observed correlation did not appear by chance. This example illustrates the distinction between the two basic computational goals of covariation-based causal induction (Griffiths \& Tenenbaum, 2005). Causal structure induction refers to the use of covariation information to decide whether or not a causal link exists in the world, without regard to the strength of that causal link. Parameter estimation (also called parameterization and strength induction), on the other hand, refers to the use of covariation to quantify how strongly causes relate to effects-namely, to predict with some accuracy the probability of an effect given the presence or absence of its causes. In the headache example, the person may be quite confident that a causal link exists but be in a state of considerable uncertainty about the strength of that link. Many models of causal judgment run these separate beliefs together and therefore risk failing to preserve an important normative distinction.

\section{A Graphical Model for Simple Causal Tasks}

In the past decade, a number of researchers from fields related to formal computation have developed sophisticated ways to represent causal structure. Graphical models (also called Bayesian causal models or causal Bayes nets) represent variables in the world as nodes, and causal relations between those variables as arrows connecting nodes directionally: An arrow connecting Node A to Node B indicates that the variable represented by Node $\mathrm{A}$ has a causal influence on the variable represented by Node B. For example, if two switches are connected to a bulb, either, neither, or both of them may cause the bulb to illuminate. This can be represented graphically as a net with three nodes and two links. In both cases, the two nodes representing the possible states of the two switches would have direct links to a single node representing the possible states of the bulb. To be precise, the nodes represent the three variables that define the possible values (in this case, on or off ) of the switches and the bulb, and the links specify that the value of the variable representing the bulb is determined exclusively by the values of the variables 
representing the two switches. This kind of representation is moot about how the causes might interact (see below).

A graphical model implements the idea that the state of any variable can be revealed by knowing the state of each of the variables with links to it (its parents, if we refer to nodes, instead of variables) and the parameters defining those links. The key assumption (Griffiths \& Tenenbaum, 2005 , p. 345) is called the Markov condition, and it computationally implements the restriction that the probability of a node's being in a particular state is the product, across all of the node's parents, of the probability of its being in that state given each of its parents.

Imagine a causal problem resulting from a virus-induced disease in which a reasoner is provided information on a number of patients: Some of them have the virus and develop the disease (type $\boldsymbol{a}$ events), some have the virus but do not develop the disease (type $\boldsymbol{b}$ ), some do not have the virus but suffer the disease (type $c$ ), and finally, some neither have the virus nor suffer the disease (type $\boldsymbol{d}$ ). Two nodes would stand for two variables representing the presence/absence of the virus (the potential cause, $C$ ) and of the disease (the effect, $E$ ), and a causal arrow is hypothesized to exist between them $(C \rightarrow E)$. Each of the variables can adopt two values representing the presence or the absence of the cue $C\{c, \sim c\}$ and the effect $E\{e, \sim e\}$. Henceforth, we will simplify $P(C=c), p(C=\sim c), P(E=e)$, and $P(E=$ $\sim e$ ) as $P(C), P(\sim C), P(E)$, and $P(\sim E)$; the same notation will be adopted for conditional probabilities, and, for the sake of coherence, we will use uppercase letters to denote the causes and the effect as events. Since there are no other explicit causes, it is also necessary to assume that some hidden cause or set of causes generates the outcome when the cue is absent (in $c$ type trials). That background cause, unknown to the reasoner, can be represented in the model as a second parent of the outcome $A\{a, \sim a\} .{ }^{1}$ The resulting graphical model can thus be represented as $C \rightarrow E \leftarrow A$. But there is another potential model, identical to the first but without the link between $C$ and $E$. The covariation data may be more consistent with either model.

Suppose participants are asked to judge how intense or strong the causal relationship between the candidate cause $(C)$ and the effect $(E)$ is. This request can be interpreted in two ways: first, whether the available information is sufficient to infer that there is a causal link (structure); and second, what the likelihood is that the virus would cause the disease in a given circumstance (parameterization/strength). In general terms, in relation to the first question, the higher the contingency and the larger the sample of patients observed, the more certain the participants will be that the virus, and not other hidden causes in the environment, is the true cause of the disease (put differently, that the model virus $\rightarrow$ disease $\leftarrow$ other causes is more appropriate than the simpler model disease $\leftarrow$ other causes). In relation to the second question, if the causal link between the virus and the disease is assumed to exist, the higher the contingency, the stronger the influence of the virus on the disease.

In addition to deciding what the strength of the relationship between $C$ and $E$ might be (assuming that a causal link does exist between them), a further problem is to decide how they functionally interact (Danks, 2002; Glymour, Scheines, Spirtes, \& Kelly, 1987; Griffiths \& Tenenbaum, 2005; Pearl, 2000; Perales \& Catena, 2006; Steyvers, Tenenbaum, Wagenmakers, \& Blum, 2003). It may be the case that $C$ simply increases the probability of $E$ in a linear fashion, regardless of the base-rate probability of $E$. Alternatively, $C$ might interact with the background causes $A$ to produce the effect. For example, on an occasion when $A$ is insufficient to produce $E$, the introduction of $C$ might cause $E$, but on another occasion when $A$ is already sufficient to cause $E$, the introduction of $C$ will have no further causal impact because $E$ cannot be produced twice. The nature of the functional interaction of the potential causes is not reflected in the causal structure itself but rather in the parameterization of the graph (which, as noted above, also includes placing a value on the strength of the causal links).

It might seem that causal learning, according to this normative framework, represents an insoluble chickenand-egg problem. Even if a particular form is assumed for the interaction between $C$ and $A$, it remains the case that the strength of the links cannot be determined until the structure has been decided, while at the same time the decision about structure depends on the weights that are assigned to the links. The major contribution of Griffiths and Tenenbaum (2005) is to have solved this problem by specifying the calculation of support (described in more detail below) - that is, the evidence that the contingency data favor one model over another-without committing to particular values for the strength of the links. This is achieved by integrating the evidential support across all possible strength values.

One important aspect of graphical modeling is its usefulness as a language for representation. Graphs are powerful tools for representing the causal structure of the world. Another aspect is causal induction. A number of artificial intelligence (AI) algorithms has been proposed to induce causal graphs (or causal structure), and parameterize them, from patterns of co-occurrence or covariation among variables in the world (Pearl, 2000; Spirtes, Glymour, \& Scheines, 1998). To date, no one has explicitly proposed that naive reasoners represent complex causal structures in the form of graphs (or some psychological translation of them; see Glymour, 1998) or carry out induction processes as AI algorithms do. However, the distinction between parameterization and structure is more or less explicit in at least two psychological models of causal induction (power and support). Next, we consider three psychological models of causal induction directly inspired by normative principles. $\Delta P$ (or contingency), power, and support are interpreted in terms of the parameterizationstructure distinction.

\section{Norm-Based Models of Human Covariation-Based Causal Induction}

\section{Contingency}

The most frequently cited statistic for measuring the degree to which a candidate cause and an effect are related is $\Delta P$, an index of the predictive value of the cause computed 
as the difference between the probability of the effect in the presence of the cause and the probability of the effect in the absence of the cause:

$$
\Delta P=P(E \mid C)-P(E \mid \sim C)=\boldsymbol{a} /(\boldsymbol{a}+\boldsymbol{b})-\boldsymbol{c} /(\boldsymbol{c}+\boldsymbol{d}) .
$$

In this equation and henceforth, $\boldsymbol{a}, \boldsymbol{b}, \boldsymbol{c}$, and $\boldsymbol{d}$ stand for the frequencies of the four trial types described above (see note 1$)$.

If we maintain the scheme outlined in the previous section, $\Delta P$ can be reformulated as $\Delta P=P(E \mid C \cdot A)-$ $P(E \mid \sim C \cdot A)$. The addition of $A$ indicates that the occurrence of the effect $(E)$ in the presence of the candidate cause $(C)$ is accounted for by the conjoint occurrence of the candidate cause and the set of unknown alternative causes in the background $(A)$, whereas the occurrence of the effect in the absence of the candidate cause is accounted for only by the presence of such a set of alternative causes.

Manipulations of $\Delta P$ have a significant effect on different types of causal estimates and other learning indices (see, e.g., Allan \& Jenkins, 1983; Lovibond, 2003; Wasserman, 1993). This finding originally led to the proposal that reasoners actually compute $\Delta P$ in order to derive an estimate of the strength of interevent causal relations. From a normative perspective, however, the relationship between contingency, parameterization, and structure is not simple (see Griffiths \& Tenenbaum, 2005; Tenenbaum $\&$ Griffiths, 2001). On the one hand, $\Delta P$ provides some information about structure, in that higher absolute $\Delta P$ values provide stronger evidence that a generative or preventive causal link between $C$ and $E$ actually exists. On the other hand, $\Delta P$ does not provide a measure of the causal influence of the candidate cause $(C)$ that is independent of the presence of alternative causes $(A)$. Imagine a candidate cause in a context in which the effect occurs with a low base rate (e.g., .10). In this case, $\Delta P=.20$ would mean that the introduction of the candidate cause in that context increases the probability of the effect up to .30 . Let us also imagine a second situation in which the base rate of the effect is .80 . In this case, $\Delta P=.20$ would mean that the candidate cause increases the probability of the effect to 1.00. If we assume the candidate cause and its potential causal influence to be the same in both cases, we must conclude that the introduction of the cause in a given context with a certain effect base rate simply makes that base rate increase linearly. The causal structure in this case is parameterized in such a way that the independent probabilities of the effect attributable to $C$ and $A$ summate (i.e., the so-called linear parameterization; see next section for an example and a counterexample).

It has been repeatedly demonstrated that causal judgments deviate from contingency in a number of experimental preparations (Allan \& Jenkins, 1983; Baker, Vallée-Tourangeau, \& Murphy, 2000; Lober \& Shanks, 2000; Perales \& Shanks, 2003). In light of the previous argument, however, this does not imply that judgments deviate from normativity. If the reasoner interprets the causal probe question as referring to the change in the probability of the effect produced by the candidate cause in the presence of the set of unknown causes $(A)$, then $\Delta P$ would be the correct answer. However, as noted above, unless the linear parameterization is assumed, $\Delta P$ cannot be considered the appropriate measure if the reasoner understands that he or she is being asked about the contextindependent influence of the candidate cause.

\section{Power}

Computation. Without extra assumptions, knowing the frequencies of $\boldsymbol{a}-\boldsymbol{d}$ in a contingency table is insufficient to estimate all the parameters necessary to describe the causal structure. As noted, the way in which the candidate cause and the background causes interact requires a background assumption. It has been customarily assumed that, unless contradictory evidence is provided, naive reasoners tend to adopt the simplest possible causal model.

This simplicity principle is implemented in the assumption that, by default, any two causes of the same effect will be considered to be noninteractive. To illustrate this idea, imagine two possible causes of an allergic reaction: eating shrimp and eating peanuts. Let us also imagine that, if one eats peanuts but not shrimp, the probability of the allergic reaction is .40 , and if one eats shrimp but not peanuts, the probability of the allergic reaction is .50. If one eats shrimp and peanuts, and the effects of the two causes are probabilistically independent, then $P($ allergy $\mid$ shrimp $\cdot$ peanuts $)=[P($ allergy $\mid$ shrimp $\cdot \sim$ peanuts $)+P($ allergy $\mid$ peanuts $\cdot \sim$ shrimp $)-P($ allergy $\mid$ shrimp $\cdot$ $\sim$ peanuts $)] \times P($ allergy $\mid$ peanuts $\cdot \sim$ shrimp $)=.70$. In other words, according to probability theory and assuming independence, 70 out of 100 people who eat shrimp and peanuts will suffer the allergic reaction. This way of combining two causes is customarily called (see Glymour, 1998; Pearl, 1988) the noisy-or schema (if applied to generative causes), or the noisy-and-not schema (if applied to a pair composed of a preventive and a generative cause) (see Glymour, 1998; Pearl, 1988). Note that this is quite different from the linear parameterization discussed previously, according to which the probabilities would summate to yield $P($ allergy $\mid$ shrimp $\cdot$ peanuts $)=.90$.

Although the terms noisy-or and noisy-and-not come from a separate literature (see Glymour, 1998), the power PC theory (Cheng, 1997, 2000) is actually the normative rule for estimating parameters on the basis of applying the noisy-or schema (or the noisy-and-not schema) to the graphical model outlined above for simple causal induction (see Cheng, 1997, for a detailed description of the mathematical derivations underlying the theory).

The causal power (pow) of a generative cause is defined in the power PC theory as the probability of the effect given the presence of that cause in an imaginary context in which all other possible causes of the same effect have been removed. In the case of preventive causes, causal power is defined as the decrease in the probability of the occurrence of an effect produced by the preventive cause, provided that in the absence of the cause the same effect would occur for sure.

Given that causal power refers to the influence of the candidate cause in a hypothetical, alternative, cause-free context, it cannot be directly observed. However, causal power can be inferred from observable contingencies 
as long as the noisy-or schema holds. If the conditions stemming from it are met, the causal power of a candidate cause can be calculated according to Equation 2 for generative causes and according to Equation 3 for preventive causes:

$$
\begin{aligned}
& \text { pow }=\Delta P /[1-P(E \mid \sim C)], \\
& \text { pow }=\Delta P / P(E \mid \sim C) .
\end{aligned}
$$

Therefore, computationally, the power PC theory is a model for parameter estimation (see Griffiths \& Tenenbaum, 2005). Once $P(E \mid \sim C)$ and $P(E \mid C)$ are known from observation, and the independence and simplicity assumptions (the noisy-or or the noisy-and-not schemata) are applied, causal strength can be computed. As a normative theory, on the other hand, the power PC theory falls short because it doesn't provide a mechanism for deciding what the correct causal structure is.

Problems with the application of the power PC theory to standard tasks. It has been recently claimed that causal power estimation takes place in stages and that early stages may be affected by learning, memory, and attentional constraints. The power PC theory does not make any particular assumption about how covariation and probabilities are computed. This means that the process of evidence accumulation via learning is not considered. Hence, causal judgments are compatible with the theory's predictions if and only if those judgments are made after the learning process has reached its asymptote (Lober \& Shanks, 2000; Perales \& Shanks, 2003).

If we assume that people compute contingency or conditional probabilities first (Buehner, Cheng, \& Clifford, 2003) and power second, according to Equation 2 or Equation 3, then any bias in detecting conditional probabilities would in turn affect the ensuing causal judgment. That judgment would then be biased because of an error in computing the input variables to the power calculation. In consequence, testing the power PC theory requires insuring that there is no bias in the perception of the conditional probabilities (Buehner et al., 2003), or at least taking such biases into account when drawing predictions from the model. Of course, the same argument applies to testing $\Delta P$.

It is important to note here that some recent studies (Maldonado, Jiménez, Herrera, Perales, \& Catena, 2006; Perales, Catena, Shanks, \& González, 2005) have directly addressed the issue of conditional probability and trial type frequency estimation in trial-by-trial tasks in which the procedure imposes certain memory demands on the causal inference process. Convergent data demonstrate that, typically, the only effect memory demands have on causal estimation is to increase the noisiness of judgments but not to differentially bias either con ditional probability or frequency estimates. This means that, although judgments under memory demands are expected to be slightly more imprecise, this should not alter the ability of pow to predict group mean judgments.

A further important issue is that Buehner and his collaborators (Buehner \& Cheng, 1997; Buehner et al., 2003) have argued that most observed deviations from causal power (pow) reported in the literature are due mainly to ambiguities in the probe question used. For example, in Perales and Shanks's (2003) study, the causal question was worded as follows: "Please give an estimate of the degree to which you think [the candidate cause] causes or prevents [the effect]." This example is representative of the standard type of probe question often used in simple causal induction experiments. Henceforth, we will denote as standard probe questions those in which (1) the question refers to the influence of the cause in general terms, without specifying in which context that influence takes place; (2) the judgment is expressed in the form of a quantitative value (normally on a bipolar scale, representing preventive and generative values symmetrically); and (3) the question is formulated in causal terms; that is, the term cause or causal is explicitly mentioned.

It could be the case that causal judgments are not closely calibrated to power simply because some participants interpret the probe question as requiring them to evaluate the effect of the candidate cause in the same context in which the causal relationship was learned, rather than in a hypothetical context in which alternative causes are absent. Consequently, the normative reference for that causal interpretation is not power but $\Delta P$. If reasoners are sensitive to this distinction, their judgments would be expected to deviate from pow to some degree. In order to solve this problem, it has been proposed that the question should be worded in counterfactual terms (see Buehner \& Cheng, 1997; Buehner et al., 2003). For instance, the counterfactual question in Collins and Shanks's (2006) study, in which the cover story was about the effect of a certain type of nuclear radiation on the occurrence of a mutation in a species of butterfly, was worded thus: "How many out of 100 butterflies, none of which would show a mutation if unradiated, do you estimate would show a mutation if radiated?" This wording is claimed to eliminate ambiguities in participants' interpretations, which implies that it should better measure pow than standard causal questions do. That is to say, judgments elicited with this rating question should conform better to pow than to $\Delta P$.

According to Buehner et al. (2003), standard judgments are thus a suboptimal test of the power PC theory. Outside the theory, however, there is no reason to think that counterfactual judgments are preferable for assessing reasoners' causal knowledge compared with standard judgments. Actually, some unpublished data from our own laboratory (Perales \& Shanks, 2004) show that the counterfactual probe question poses even more interpretational problems for the theory than do standard ones. Instead of conforming to pow, judgments elicited with counterfactual questions were driven very heavily by $P(E \mid C)$, a strategy earlier identified by Anderson and Sheu (1995). For instance, judgments differed radically in a pair of conditions equal in pow but differing in $P(E \mid C)$.

In any case, that controversy is not the focus of the present review. The fact that the theory proposes a particular measure as the most apt to test its predictions does not mean that those predictions cannot be tested against a more commonly used measure. And, indeed, the theory was originally formulated as an account of standard judgments (Cheng, 1997). Here, our interest is in assessing 
viable explanations of the large body of available data and in building a bridge between that corpus of data and new theoretical developments.

\section{Support}

As noted above, according to graphical models, parameter estimation and causal structure induction should be regarded as separate computational goals in human causal induction. Additionally, the psychological implementation of this assumption implies that attaining these goals requires different cognitive operations. Griffiths and Tenenbaum (2005, p. 349) have stated that "much of human causal induction seems to be directed at the problem of inferring the qualitative causal structure responsible for a set of observations." In the case of elemental causal induction, the problem of structure learning boils down to deciding whether in the $C \rightarrow E \leftarrow A$ schema, the link between $C$ and $E$ actually exists or should be removed, given a set of observations.

Following Griffiths and Tenenbaum's (2005) notation, we will denote the graph with that causal link as Graph and the graph without it as $\mathrm{Graph}_{0}$. Making a structural decision thus implies deciding which of the two graphs $\left(\mathrm{Graph}_{1}\right.$ or $\left.\mathrm{Graph}_{0}\right)$ best describes the observed data. That decision is made on the basis of the application of Bayes's rule, expressed here in log odds

$$
\begin{aligned}
\operatorname{support}\left(\operatorname{Graph}_{1}\right)= & \log \frac{P\left(\operatorname{Graph}_{1} \mid D\right)}{P\left(\operatorname{Graph}_{0} \mid D\right)} \\
= & \log \frac{P\left(D \mid \mathrm{Graph}_{1}\right)}{P\left(D \mid \mathrm{Graph}_{0}\right)} \\
& +\log \frac{P\left(\operatorname{Graph}_{1}\right)}{P\left(\mathrm{Graph}_{0}\right)}
\end{aligned}
$$

where $D$ (data) stands for the set of observations provided to the individual during the learning task. Given a certain parameterization (e.g., the noisy-or schema and power values computed according to power PC), both $P\left(D \mid \mathrm{Graph}_{1}\right)$ and $P\left(D \mid \mathrm{Graph}_{0}\right)$ are computable from the available evidence. In addition, in the absence of any previous information in favor of one model or the other, $P\left(\mathrm{Graph}_{1}\right)=P\left(\mathrm{Graph}_{0}\right)$, and thus the second term on the right side of the equation can be removed. In consequence, in order to compute support, it is necessary to compute only $P\left(\mathrm{Graph}_{1} \mid D\right)$ and $P\left(\mathrm{Graph}_{0} \mid D\right)$. Support is the log ratio between these two probabilities and can be interpreted as a measure of the relative evidence available to the reasoner in favor of one graph or the other or, what amounts to be the same thing, in favor of or against the existence of a causal link between the candidate cause and the effect. In terms of the power PC theory, support can also be interpreted as the amount of evidence that pow for the candidate cause is different from zero.

The computation of support is mathematically complex and will not be described here. However, its intuitive meaning is simple. Ultimately, it is the contrast (in the form of $\log$ odds) between evidence that confirms and evidence that disconfirms the hypothesis that a causal link exists. Critically, this calculation can be made without making any commitment about the strength of the causal connections, since support is computed by integrating across all possible strength values. According to the model, standard causal judgments depend, at least partially, on what the reasoner believes the odds of the two competing graphs to be. Thus, causal judgments are expected to mirror support. Indeed, if we analyze the customary wording for causal probe questions, there is frequently a reference to the degree to which the reasoner thinks the target cue causes the effect. It is implied in the model that the most salient feature of this type of question is the demand to make a structural judgment.

It is important to stress, however, that the type of structural decision that support is supposed to underlie requires an explicit assumption about how the target cause and the background combine with each other to produce the effect. The specific value resulting from Equation 4 will obey Griffiths and Tenenbaum's (2005) predictions if and only if the noisy-or schema is assumed (that is, if pow is used to compute causal power) - not if other models of cause interaction determine structure parameterization. Thus, as they stand at the moment, the power PC and the structurelearning models form an interlinked theoretical set that derives from and conforms to normative principles.

\section{Nonnormative (Algorithmic) Models of Human Covariation-Based Causal Induction}

A number of algorithmic models are also compatible to some degree with the data pattern shown by standard causal judgments, a pattern that coincides, for the most part, with the data pattern shown by contingency judgments (for reviews, see Allan, 1993, 2003; De Houwer \& Beckers, 2002). Note that assessing algorithmic models implies temporarily abandoning the distinction between parameter estimation and structure learning, since this distinction is not adopted by these models. Later in this article, we will evaluate the merits of the winning algorithmic model in the light of this distinction.

Two general types of algorithmic models have been proposed to account for the way in which covariational information is used for causal estimation (see Allan, 1993; De Houwer \& Beckers, 2002; Shanks et al., 1996): rulebased and associative models.

Rule-based models assume that reasoners keep track of the frequencies of the different types of trials or the conditional probabilities that they are exposed to during the task and combine those probabilities or frequencies according to a given rule or statistic. The two most frequently cited rules of this sort are $\Delta P$ and $\Delta D$ (or some other variation of the linear combination rule; see Schustack \& Sternberg, 1981). We earlier classified $\Delta P$ as a normative model. However, given its inability to account for well-established biases, some authors have considered the possibility that probabilistic contrasts are calculated on the basis of weighted probabilities (see Allan, 1993; Anderson \& Sheu, 1995). In other words, causal judgments are based on the contrast between the two conditional probabilities $P(E \mid C)$ and $P(E \mid \sim C)$ but those probabilities are not attributed the same relevance. Typically, the weight 
assigned to $P(E \mid C)$ would be larger than the weight assigned to $P(E \mid \sim C)$. Such a weighted rule could no longer claim to be normative, hence its inclusion in the present section. Regardless of its potential normative value, $\Delta P$ has been repeatedly proposed to be the rule people employ to incorporate covariational information into causal relationships (Cheng \& Holyoak, 1995; Cheng \& Novick, 1992; Waldmann, 2000).

$\Delta D$ is a simplification of the linear combination rule (from the four trial type frequencies), consisting of a mere contrast between the diagonal sums of the contingency table $[(\boldsymbol{a}+\boldsymbol{d})-(\boldsymbol{b}+\boldsymbol{c})]$. The psychological justification for such a rule, as initially proposed by Inhelder and Piaget (1958), is rather simple: Reasoners assess the amount of evidence that they consider to confirm the existence of a generative causal link-or to disconfirm the existence of a preventive link - against the amount of evidence that disconfirms the existence of a generative link-or that confirms the existence of a preventive link (note that the notion of support is very close to this concept). However, the original formulation of this heuristic has important shortcomings. First, it does not account for differential trial type weighting, and second, its value depends linearly on the number of observations, which is contradicted by evidence showing that causal judgments tend to be stable once an asymptotic learning level is reached. In consequence, the original rule has been modified in several significant aspects. In all cases, the nonprobabilistic and heuristic nature of the original rule still holds. Here, we will adopt a slightly modified version of the one proposed by Busemeyer (1991). Henceforth, we will refer to this model as the evidence integration (EI) rule:

$$
\begin{aligned}
\mathrm{EI}= & \frac{w_{a} \boldsymbol{a}+w_{d} \boldsymbol{d}}{w_{a} \boldsymbol{a}+w_{b} \boldsymbol{b}+w_{c} \boldsymbol{c}+w_{d} \boldsymbol{d}} \\
& -\frac{w_{b} \boldsymbol{b}+w_{c} \boldsymbol{c}}{w_{a} \boldsymbol{a}+w_{b} \boldsymbol{b}+w_{c} \boldsymbol{c}+w_{d} \boldsymbol{d}},
\end{aligned}
$$

where $w_{a}, w_{b}, w_{c}$, and $w_{d}$ are the weights assigned to each trial type. Despite its apparent complexity, the intuitive meaning of this equation is rather simple: People contrast the proportion of confirmatory evidence against the proportion of disconfirmatory evidence and attribute different evidential values to the four cells of the contingency matrix accordingly. Thus, EI qualitatively subsumes unweighted heuristics (in which all weights $=1$ ) and linear heuristics in which trial types are completely disregarded (e.g., the $\boldsymbol{a}-\boldsymbol{b}$ and the $\boldsymbol{a}-\boldsymbol{c}$ rules; see Allan, 1993).

Weights can reflect memory or attentional factors affecting the coding of frequencies during the training phase (Maldonado et al., 2006) or the strategy adopted by the reasoner at test (White, 2003a, 2003b). According to the $p$ CI model (White, 2003c), the specific formulation of the algorithm can vary from individual to individual, depending on which instances are considered confirmatory and which disconfirmatory in relation to a previous generative or preventive hypothesis.

There are other implementations of the idea that people contrast confirmatory and disconfirmatory evidence ad- ditively when making a judgment. For example, the linear combination rule (Anderson \& Sheu, 1995; Schustack \& Sternberg, 1981) uses a nonnormalized additive combination of cell frequencies, or the linear regression equation of judgments over cell frequencies, to fit judgments; weighted $\Delta D$ (Catena, Maldonado, \& Cándido, 1998) proposes a modification of Equation 5 in which weights are removed from the denominator. Predictions based on these versions are qualitatively similar to those based on the rule analyzed here ${ }^{2}$ (although see White, 2004).

Associative models, on the other hand, assume that causal links are learned, in the same way that other associations are, by means of a mechanism that accumulates associative strength in the link between the mental representations of the cue (or cause) and the outcome (or effect) (Shanks, 1995; Shanks \& Dickinson, 1987). The most widely known associative model is the Rescorla-Wagner rule (RW; Rescorla \& Wagner, 1972):

$$
\Delta V_{i}=\alpha \beta\left(\lambda-\Sigma V_{T}\right)
$$

According to this model, the increment in the associative strength between a cue and an outcome $\left(\Delta V_{i}\right)$ is a multiplicative function of the salience of the cue and the outcome ( $\alpha$ and $\beta$ ), and the difference between the maximum associative strength an outcome can recruit ( $\lambda$, the asymptotic learning level) and the associative strength recruited by all the cues present at the current trial $\left(\Sigma V_{T}\right)$. The increment in associative strength on a given trial is a function of the degree to which the outcome is unpredicted by the cues presented on that trial. $\alpha$ and $\beta$ are assumed to take values between 0 and $1 ; \lambda=1$ on those trials in which the outcome is present and 0 on trials in which it is absent. An absent cue cannot recruit associative strength, although other cues present on those trials (e.g., the context) can, and these compete with the target cue for associative strength (determined by $\lambda$ ).

If the RW rule is restricted in such a way that $\beta$ always takes the same value (independently of whether the outcome is present or absent in the current trial; $\beta_{\mathrm{O}}=\beta_{\sim \mathrm{O}}$ ), its predictions are equal to $\Delta P$ at asymptote (Chapman \& Robbins, 1990). Therefore, this restricted version of RW can be rejected on the same empirical basis as $\Delta P$. The unrestricted version does not fit the pattern of results either, since it requires a given parameter order $\left(\beta_{\mathrm{O}}<\beta_{\sim \mathrm{O}}\right)$ to account for the positive effect of pow on estimates when $\Delta P$ is held constant in positive contingency tasks and the opposite order of parameters $\left(\beta_{\mathrm{O}}>\beta_{\sim \mathrm{O}}\right)$ to account for the effect of the same manipulation on estimates in negative contingency tasks. Although that change could be justified in comparisons across experiments (Lober \& Shanks, 2000), it is not justified when negative and positive contingencies are included in the same experiment with the same cover story and the same materials for all conditions (Perales \& Shanks, 2003).

Several modifications of the original RW model have been proposed to address its shortcomings. As we have shown elsewhere (Perales \& Shanks, 2003), Van-Hamme and Wasserman's rule (1994) and the RW model formally make the same asymptotic predictions in simple one-cause, 
one-effect tasks. Another model, SOP (sometimes opponent processes; see Aitken, Larkin, \& Dickinson, 2000, 2001; Dickinson \& Burke, 1996), is a real-time model, which means that it would be computationally too demanding to test with the simulation procedure we have used here. But in any case, if we hold temporal parameters constant, it is an error-reducing rule functionally identical to RW, and hence parameter optimization should lead to identical predictions, making predictions for one-cue, one-outcome trial-by-trial tasks that are virtually indistinguishable from those made by the RW rule (they were formulated to deal with order and cue competition effects the original RW rule had difficulties dealing with). Adjusting parameter sets to the best fit, the Van-Hamme and Wasserman, SOP, and RW models make indistinguishable quantitative predictions for one-cause, one-effect simple tasks (see Perales $\&$ Shanks, 2003). So, henceforth, we will denote the three models with the single label RW.

Pearce's (1987) model is also an elaboration of the original RW rule. This model is based on the assumption that any set of cues presented during the task is represented as a single configuration and recruits associative strength as such. Additionally, when a configural cue is presented, it not only activates its own representation but also the representations of similar configurations that have also been presented during the task. In one-cue, one-outcome tasks, two configurations are present: one comprising contextual cues $(X)$ and a second comprising the target cause and the contextual cues in the background $(C X)$. When one of these configurations is presented, the representation of the other is also activated by virtue of the similarity between them, and therefore,

$$
\Delta V_{C X}=\beta\left(\lambda-V_{C X}-x_{1} V_{X}\right)
$$

and

$$
\Delta V_{X}=\beta\left(\lambda-V_{X}-x_{2} V_{C X}\right)
$$

Equation 7 applies on those trials in which the candidate cause is present ( $\boldsymbol{a}$ and $\boldsymbol{b}$ ), whereas Equation 8 applies on those trials in which the cause is absent $(\boldsymbol{c}$ and $\boldsymbol{d})$. All of the other parameters have the same meaning as in the RW rule. The two new parameters, $x_{1}$ and $x_{2}$, represent the degree of generalization from $X$ to $C X$ and vice versa, ${ }^{3}$ and their value is determined by the degree of similarity between them. Finally, the judgment of the candidate cause $(C)$ is determined by the similarity $\left(x_{3}\right)$ between $C X$ and $C$, according to the following expression:

$$
J_{C}=x_{3} V_{C X} \text {. }
$$

As Perales and Shanks (2003) also showed, asymptotic predictions of Pearce's (1987) model do not depend on $\beta$. We have also carried out additional simulations with a trial-by-trial implementation of the model, including the possibility that $\beta$ takes different values for $X$ and $C X$. There were no cases in which these modifications altered the qualitative pattern of judgments predicted by the model, and, thus, the asymptotic version of Pearce's model elaborated by Perales and Shanks (2003, Appendix 2) was adopted for all the simulations reported in the remainder of this article.

\section{The Search for the Best-Fitting Model for Standard Judgments in Trial-by-Trial Experiments}

In trying to evaluate the merits of the several available models, we exploited the fact that there is a massive amount of data, obtained using standard judgments, that still remains to be comprehensively accounted for. We report a meta-analysis that highlights the regularities contained in this rich data set.

\section{Study Selection}

For testing, we considered all of the experiments carried out to date in which (1) single interevent causal relations were assessed over a constant background; (2) covariational information was presented trial by trial, since associative models are learning based and thus only applicable to this type of presentation format; (3) standard causal questions were used (in which simply a general estimate of the relationship, and not a specification of the context in which the question applied, was required); (4) global ratings, rather than trial-by-trial adjustment ratings, were elicited from participants; and (5) no other manipulations were carried out apart from the frequencies of the four trial types. To our knowledge, the following studies fulfill these criteria: Wasserman, Kao, Van-Hamme, Katagiri, and Young (1996, Experiment 1); Vallée-Tourangeau, Murphy, Drew, and Baker (1998); Lober and Shanks (2000); Shanks (2002); Buehner et al. (2003, Experiment 3); Perales and Shanks (2003); White (2003c, Experiments 2 and 6); and Collins and Shanks (2006). Note that, because of the number of conditions, the studies by Vallée-Tourangeau et al. (1998), Buehner

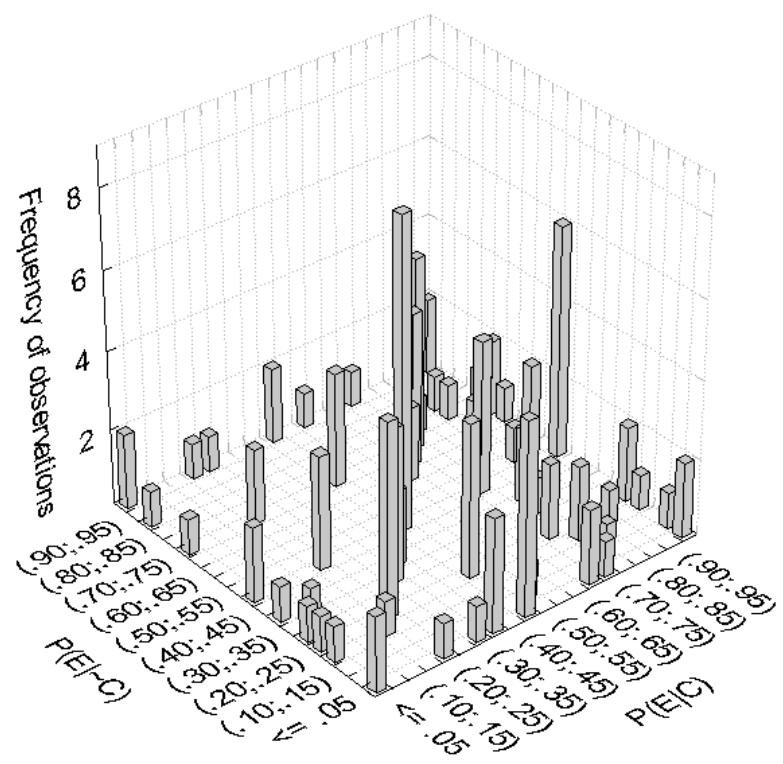

Figure 1. Frequency of observations per $P(E \mid C)$ and $P(E \mid \sim C)$ combination in the selection of experiments in the meta-analysis. The figure shows a broad sampling of the contingency space, with a tendency for greater representation of low and moderately positive contingencies. 
et al. (2003), White (2003c), and Wasserman et al. (1996) are especially representative. Although some studies from our own laboratory fulfill the criteria, they comprise a small set of conditions, and their global influence is modest (see the Appendix). The 114 conditions are fairly well spread across the contingency space, as shown in Figure 1. Some $P(E \mid C)$ and $P(E \mid \sim C)$ combinations, corresponding to low and moderately positive contingency values, are slightly overrepresented (which is not necessarily problematic for model fitting). More importantly, the whole contingency space is covered by the selected studies.

Anderson and Sheu's (1995) Experiment 1 was not included for two reasons. First, some conditions in that experiment comprised only four trials, which leaves open the possibility that final judgments were preasymptotic; and second, that experiment alone included 80 different experimental conditions. If all of these conditions were considered, our data would clearly depend excessively on a single experiment. On the other hand, we decided to include Vallée-Tourangeau et al.'s (1998) study. In spite of the fact that participants in that study's experiments were presented with two candidate causes, one of the causes was held constantly present and can hence be considered to be similar to a background cause.

The two experiments in the Appendix attributed to Collins and Shanks (2006) actually belong to an earlier, unpublished version of that article (the published version included only Experiment 1). In addition, we have included in the analyses an unpublished experiment from our laboratory (noted in the Appendix as Perales \& Shanks, 2004, Experiment 1A). We checked that neither the exclusion of
Anderson and Sheu's (1995) experiment nor the inclusion of Vallée-Torangeau et al.'s (1998) and our experiments made any significant difference with regard to the conclusions of the present study.

The mean judgments observed in each of the conditions in the previously listed set of experiments are shown in the Appendix. In order to facilitate the interpretation of that table, we have graphed an idealized representation (a smoothed scatterplot) of the causal judgments, plotted in relation to $P(E \mid C)$ and $P(E \mid \sim C)$. The result is a regular surface (Figure 2, left panel) in which judgments are shown to vary more strongly as a function of $P(E \mid C)$ than as a function of $P(E \mid \sim C)$.

\section{Model Validation I: Quantitative Fit}

In this section, our first aim was to derive quantitative predictions from four candidate algorithmic models (EI, weighted $\Delta P$, Pearce, and RW) over the 114 experimental conditions included in the test series of studies (Appendix) and obtain a minimum prediction-judgment discrepancy measure (across the set of possible parameters of each model) in order to assess the goodness of each model's quantitative fit.

A quantitative comparison cannot be undertaken in a direct manner since the models differ in complexity. This means that if a simple prediction-judgment averageddiscrepancy measure were obtained for each model, that measure would tend to favor the most complex model, normally the one with a larger number of parameters. To ameliorate this problem (see Myung, Foster, \& Browne, 2000 , for a review), we have used a variant of the cross-
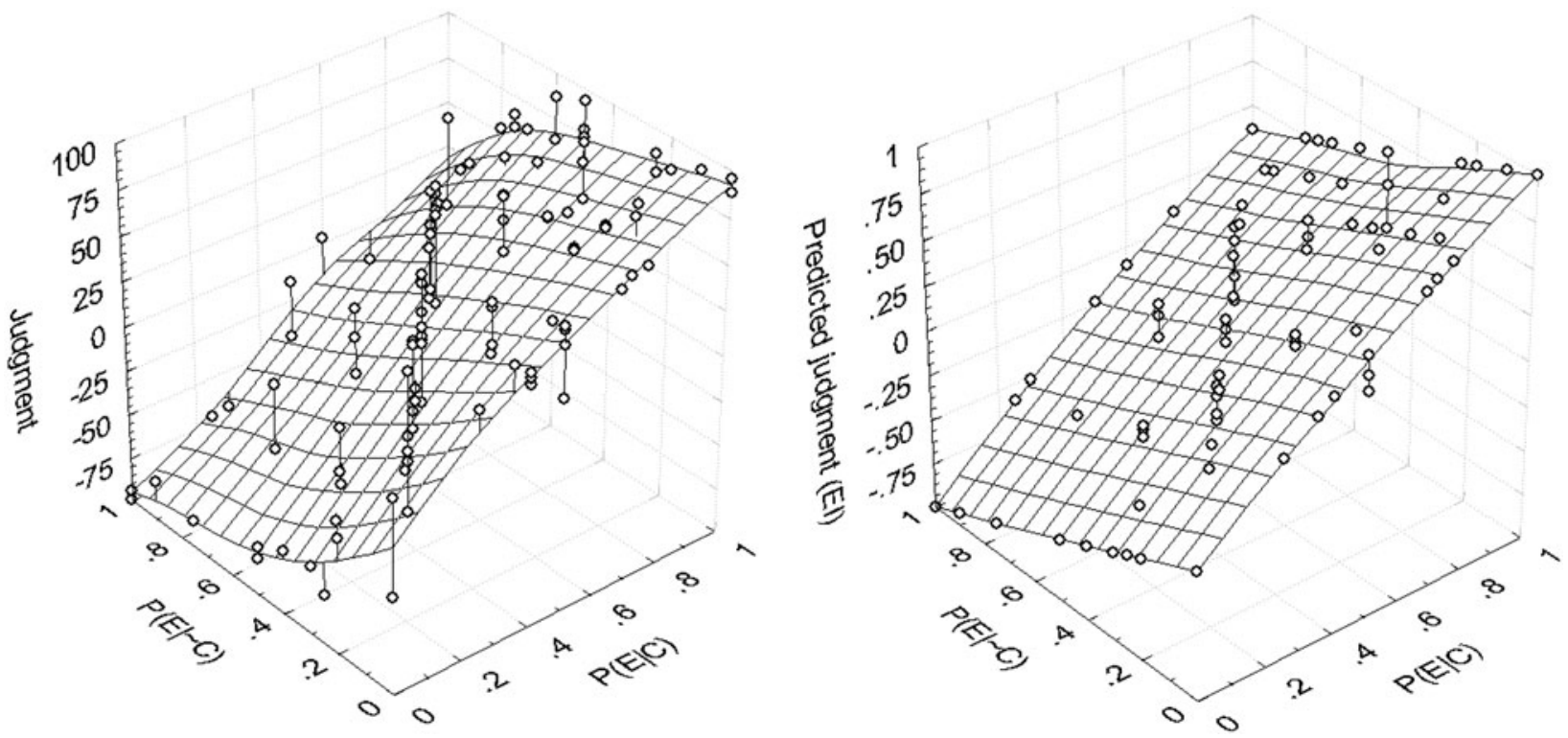

Figure 2. Left panel-smoothed scatterplots (least-square smooth routine, as implemented in Statistica '99 software) of actual judgments (as shown in the Appendix). Right panel—standard causal judgments predicted by the EI rule as a function of $P(E \mid C)$ and $P(E \mid \sim C)$. In the left panel, each point represents an observed causal estimate for a condition with the corresponding combination of conditional probabilities. In the right panel, each point represents a predicted judgment for a condition with the corresponding combination of conditional probabilities (note that there can be more than one prediction for each combination, since the same combination of conditional probabilities can result from different combinations of trial type frequencies and can thus yield different predicted judgments). The parameters used for predictions are the ones reported in the Appendix. 
validation method. In this procedure, the whole sample of $N$ observed judgments was randomly divided into two subsamples. The calibration subsample (composed of N/2 observations) was used to find a set of best-fitting parameters for each model. A Visual Basic 6.0 program explored the parameter space by carrying out a grid search with steps of size .05 between consecutive parameter values. For each model, the combination of parameters that minimized the mean of the squared difference between each prediction (multiplied by 100) and observed judgment (translated into a $-100-+100$ scale) for each experimental condition was selected (henceforth, we will denote this discrepancy measure as the least mean square error, LMSE).

Subsequently, the selected set of parameters (the parameters that minimized the discrepancy between predictions and judgments) was used to fit the pattern observed in the validation subsample (composed of the remaining $N / 2 \mathrm{ob}-$ servations in the sample). In other words, the best-fitting parameters obtained in the calibration stage were used to make predictions for the conditions not included in the calibration sample. The purpose of doing so was to fix the parameters of the model and force it to apply that same set of parameters to a different sample of data and obtain a new discrepancy measure (an LMSE measure for the $N / 2$ observations included in the validation sample) that was not dependent on the complexity of the model. Each calibration-validation step was iterated 1,000 times and the discrepancy measures for the validation subsample obtained across iterations were averaged in order to provide a global-fitting measure for each model (henceforth, average $L M S E$, or $a L M S E$ ). Although it is conceivable that the parameters might have varied systematically depending on experimental conditions, we assumed that they were invariant across experiments and conditions.

The data points used for the goodness-of-fit evaluation are those included in the Appendix. Therefore, in each iterative step, the calibration sample was composed of 57 (randomly selected) experimental observations and the validation sample of the remaining 57 observations. Beforehand, and with the aim of setting a comparison benchmark, a mean square error (MSE) was calculated for causal power and contingency, which are parameter free. The MSE obtained by averaging the squared difference between each power value and each observed judgment was 533 . The same procedure was employed with $\Delta P$, and in this case, the MSE was 528. These values can be interpreted as the average squared discrepancy between actual judgments and the standard established by pow and $\Delta P$, and they are expressed in the same units as aLMSE.

In the case of support, values are not expressed on the same scale (since support can vary from positive to negative infinity). Support values for each condition are also reported in the Appendix. Two values are reported for each condition, corresponding to parameterizations of the causal structure with the noisy-or and the noisy-and-not schemas, respectively. The first value corresponds to the relative evidence supporting the existence of a generative causal link between the cause and the effect, whereas the second value represents the evidence in favor of the existence of a preventive link between them. In cases in which contingency is positive and the a priori hypothesis that the reasoner tries to test is also positive, the noisy-or parameterization is selected for fitting. Similarly, when both the previous hypothesis and contingency are preventive, the noisy-and-not schema must be applied. However, it is not that clear what must be done when contingency is zero, especially if the previous instructions do not bias the reasoner to test a preventive or generative link. For the purpose of fitting, null contingency conditions have been considered preventive in the one case in which instructions biased the participants to think so (Buehner et al., 2003; preventive component), and generative in all other cases. In any event, support is expressed in log odds and is not easily translatable to the same scale on which the judgments are expressed (and thus, any LMSE value obtained for support-judgment fitting would be noninterpretable); most importantly, a negative generative support value does not mean the same thing as a positive preventive support value. Therefore, at this point, reported support values must be regarded exclusively as an index of the qualitative ordering of conditions predicted by the structure-learning model.

The EI rule $\left(a L M S E=306.20, w_{a}=.84, w_{b}=.58\right.$, $w_{c}=.39$, and $w_{d}=.33$ ) was the best-fitting algorithmic model, followed by Pearce's model ( $a L M S E=402.55$, $\left.x_{1}=.82, x_{2}=.40, x_{3}=.77\right)$, weighted $\triangle P(a L M S E=$ $\left.436.31, w_{1}=1, w_{2}=.81\right)$, and RW $(a L M S E=536.32$, $\beta_{\mathrm{O}}=.57 ; \beta_{\sim \mathrm{O}}=.66$ ). Independent nonparametric tests showed significant differences in $L M S E$ values, across the 1,000 iterations, between the EI rule and all the other models: Pearce's model (Mann-Whitney's $U=104,872$, $z=-30.60)$, weighted $\Delta P(U=57,432, z=-34.27)$, and RW $(U=3,195, z=-38.47)$ (nonparametric tests were used because of the systematic right-skewed shape of the LMSE distributions). The Appendix gives the predictions of each model, based on the average parameter values across the 1,000 iterations of the cross-validation analysis and applied to all 114 conditions across the data set. The EI model fits the data well, with a mean absolute discrepancy of about 12.30 points (median $=8.83$, mean squared difference $=274.97$ ) on the -100 to +100 rating scale for each of the 114 conditions. The EI model also gives appropriate weights to the cell frequency information $\left(w_{a}>w_{b}>w_{c}>w_{d}\right)$, as elaborated below.

Can some combination of pow and $\Delta \mathbf{P}$ fit better than EI? As noted above, according to Buehner et al. (2003), standard causal judgments deviate from pow simply because different people interpret the causal question in different ways. Reasoners who interpret the probe question as applying exclusively to the same context in which learning took place will take $\Delta P$ as the appropriate answer to that question, whereas those who interpret the probe question as applying to an imaginary generalization context in which no alternative causes are present will compute power. In addition, trial-by-trial tasks impose memory and attentional demands that make people perceive conditional probabilities in a biased manner. 
We therefore tested a model that combines power and contingency. We refer to this as the hybrid pseudonormative model (HPN),

$$
J=\gamma p o w^{\prime}+(1-\gamma) \Delta P^{\prime},
$$

where $J$ stands for the average judgment of a sample of participants after a series of trials, $\gamma$ represents the proportion of people who take power as the correct answer to the causal question (and, therefore, $1-\gamma$ represents the proportion of people who compute $\Delta P$ ), and pow $^{\prime}$ and $\Delta P^{\prime}$ stand for the subjective values of power and contingency, respectively, computed from systematically biased conditional probabilities. Those biases are calculated according to Equations 11 and 12:

$$
\begin{gathered}
\Delta P^{\prime}=w_{1} P(E \mid C)-w_{2} P(E \mid \sim C) \\
p o w^{\prime}=\Delta P^{\prime} /\left[1-w_{2} P(E \mid \sim C)\right] \\
\quad(\text { generative candidate causes) } \\
\text { pow } w^{\prime}=\Delta P^{\prime} / w_{2} P(E \mid \sim C) \\
\quad \text { (preventive candidate causes) }
\end{gathered}
$$

In these equations, $w_{1}$ and $w_{2}$ represent group biases in perceived conditional probabilities and take values between 0 and 1 . These parameters are included to incorporate strategically driven weighting but also to accommodate the possibility that probability estimation is biased due to the demands imposed by the type of task used. The resulting model includes three parameters and mathematically subsumes pow, $\Delta P$, and weighted $\Delta P$ (weighted $\Delta P$ is computed according to Equation 11).

After 1,000 iterations, cross-validation proved this model to be as predictive in quantitative terms ( $a L M S E=$ $\left.310.32, w_{1}=.93, w_{2}=.81, \gamma=.46\right)$ as the EI rule $(U=$ $479286, z=-1.604, p>.05)$. HPN also performed better than all the other rules. Therefore, any discrimination between the EI rule and the HPN model must be based on qualitative criteria.

A close inspection of the data confirms that most of the consistent trends in the ratings displayed in the Appendix are accounted for by the EI rule, whereas the observed qualitative patterns are at odds with the HPN rule's predictions in several respects. In order to demonstrate this, we calculated the predicted ranks of conditions in each experiment for both the EI and HPN rules and compared them with the actual ranks of judgments in each of the experiments. The sum of squared differences between predicted and actual ranks was 320 for the HPN model and 113 for the EI rule (parameters for both models were mean best-fitting parameters across the calibration samples). A two-tailed $t$ test for independent samples with unequal variances computed across the experiments in the sample showed this difference to be significant $(p=.01)$.

\section{Model Validation II: Qualitative Fit}

We have implicitly assumed that model predictions are linearly translatable into predicted judgments. However, as noted by Griffiths and Tenenbaum (2005), the relationship between judgments and the output of the cognitive algorithm underlying them could be nonlinear. ${ }^{4}$ In principle, once model complexity is controlled for, nonlinear- ity of the prediction-judgment mapping should affect all algorithmic models equally, and there is therefore no reason to assume that one or another would be differentially favored (with the exception of support, which has been excluded from the quantitative goodness-of-fit analysis). The adequacy of the analysis we have carried out is indeed reinforced by the fact that rating-prediction covariations $\left(R^{2}\right)$ reflect the same ordering of model fits as does crossvalidation (see the Appendix, bottom row).

In any case, the quantitative-fit analysis must be complemented with a more detailed qualitative analysis. Our first task is thus to identify consistent trends in the data and determine whether each model can predict such trends. The factors to be considered will be the conditional probabilities $P(E \mid C)$ and $P(E \mid \sim C)$, the base rates of the cause and the effect $P(C)$ and $P(E)$, and the frequencies of the four trial types $(\boldsymbol{a}, \boldsymbol{b}, \boldsymbol{c}$, and $\boldsymbol{d})$.

Effects of $\boldsymbol{P}(\boldsymbol{E} \mid \boldsymbol{C})$ and $\boldsymbol{P}(\boldsymbol{E} \mid \sim \boldsymbol{C})$. With other factors held constant, $P(E \mid C)$ positively relates to judgments, whereas $P(E \mid \sim C)$ relates negatively. This can be seen in virtually all of the experiments shown in the Appendix. A 3 -D depiction of the combined effects of the two conditional probabilities is displayed in Figure 2 (left panel). In addition, as Figure 2 also shows, the effect of $P(E \mid C)$ is larger than the effect of $P(E \mid \sim C)$. This effect is customarily called the sufficiency bias (Mandel \& Lehman, 1998) and is easily accounted for by the EI rule (assuming an appropriate choice of weights; see Figure 2, right panel), Pearce's (1987) model, weighted $\Delta P$ (also assuming appropriate weights), the power PC theory (and consequently also the HPN model), and the structure-learning model (support). With constraint on its parameters $\left(\beta_{\mathrm{O}}<\beta_{\sim \mathrm{O}}\right)$, the RW model accounts for the sufficiency bias in positive contingency conditions but not in negative ones. With the opposite set of parameters $\left(\beta_{\mathrm{O}}>\beta_{\sim \mathrm{O}}\right)$, the model accounts for the sufficiency bias in negative contingency conditions but not in positive ones (see Lober \& Shanks, 2000; Perales \& Shanks, 2003). Note that best-fitting parameters for RW (see quantitative fit section) obey the $\beta_{\mathrm{O}}<\beta_{\sim \mathrm{O}}$ inequality, which is probably because of the higher proportion of positive contingency conditions in the testing sample. In Perales and Shanks's (2003) Experiment 1 (see the Appendix), for example, this inequality yielded good predictions for Conditions 1 and 2 (positive contingency) but a pattern opposite to the one observed for Conditions 3 and 4 (negative contingency).

Unweighted $\Delta P$ predicts, of course, that equal variations in $P(E \mid C)$ and $P(E \mid \sim C)$ will have equal effects on judgments, contrary to what is revealed in Figure 2.

Effects of $P(E)$ and $P(C)$. The second trend worthy of attention is the outcome density effect, also called the frequency illusion. Holding contingency constant, judgments tend to vary as a function of the probability of the effect, $P(E)$, although the strength of this effect can depend on its interaction with other factors (see Griffiths \& Tenenbaum, 2005). This effect, evident in many of the studies in the meta-analysis, ${ }^{5}$ is accounted for by Pearce's (1987) model, weighted $\Delta P$ (and thus HPN), support, the EI rule, and, again, only partially by RW (as with the sufficiency bias, a single set of parameters can account for the den- 
sity bias either in positive contingency conditions or in negative ones, but not in both). On the other hand, in the subset of conditions where $\Delta P=0$, both power and RW predictions equal zero. Therefore, no effects of outcome density are predicted by either of these models in null contingency conditions. Nevertheless, these variations occur; see White's (2003c) Experiment 6 and Vallée-Tourangeau et al.'s (1998) Experiments 1 and 2.

Another pervasive trend is the effect of manipulating $P(C)$ while holding conditional probabilities constant (the cause density effect). This effect does not appear in every case, and, in global terms, its magnitude is modest. Perhaps that is why the cause density effect has remained largely underappreciated to date, although, as discussed below, it allows a clear-cut discrimination between the Pearce (1987) model, weighted $\Delta P$, and the HPN model, on the one hand, and the EI rule and support, on the other. For example, in White's (2003c) study (Conditions 1, 3, and 5 in Experiment 2), $P(E \mid C)$ and $P(E \mid \sim C)$ were held constant at 0.1 and 0.5 , respectively, whereas $P(C)$ was manipulated across three levels $(0.17,0.33$, and 0.5$)$. Judgments for those three conditions were 40, 60, and 74, respectively. For the experiments included in this metaanalysis (see the Appendix), the effect of cause density across conditions in which both $P(E \mid C)$ and $P(E \mid \sim C)$ were held constant shows up neatly in the comparisons involving the following conditions: from White (2003c), Conditions 1, 3, and 5 in Experiment 2, and 2 and 4 in Experiment 6; from Wasserman et al. (1996), Conditions 10 and 12, 8 and 13,14 and 19, and 15 and 18. In addition, we have independently replicated the cause density effect in an experiment with a very similar procedure (Perales et al., 2005). Still, the effect shows up more faintly or does not appear at all in comparisons involving the following conditions: from White (2003c), Conditions 2, 4, and 6 in Experiment 2, and 1 and 3 in Experiment 6; from Wasserman et al., Conditions 1, 2, and 5, 6 and 11, 7 and 9, and 16 and 21.

As the Appendix shows, the EI rule predicts fairly well the comparisons in which the cause density effect should appear (the proportion of variance for the observed judgments in this subset of conditions accounted for by EIpredicted judgments is $R^{2}=.76$ ).

White (2004) published a detailed report on the effect of cause density manipulations in which the effect was replicated; its interactions with other manipulations are described-particularly, the interaction between $P(C)$ and $P(E \mid C)$ across conditions in which both the total number of trials and contingency were held constant. Unfortunately, a global presentation format was adopted in those experiments, and, in consequence, the observed results do not meet the criteria to be included in this meta-analysis. However, it is important to note that augmenting the density of the cause does not have a direct effect on judgments in every case; the effect of cause density depends on the specific distribution of frequencies across the four cells of the contingency table, and it is this distribution that determines whether cause density is either directly or inversely related to judgments. Describing the precise interactions goes beyond the aims of the present article, since we are interested in the existence of such an effect rather than its sign or magnitude. Still, several instances of the cause density manipulation and its effect on both predicted and actual judgments are illustrated in the Appendix. A systematic exploration of the cause density effect in experiments with trial-by-trial presentations is overdue.

Predictions from Pearce's (1987) model, weighted $\Delta P$, and the HPN model are not affected by manipulating the ratio between the number of trials in which the cause is present and the number in which it is absent. This manipulation changes the trial type proportions without altering $P(E \mid C)$ and $P(E \mid \sim C)$, and therefore models that are based directly on conditional probabilities (HPN, power PC, weighted $\Delta P$ ), or models that are asymptotically equivalent to them (Pearce; see also Perales \& Shanks, 2003), are not able to account for any effect of this manipulation.

Pearce's (1987) model and RW predict a preasymptotic effect of cause density, but that effect is expected to disappear as learning reaches asymptote. For example, according to Pearce's model, a higher proportion of causepresent trials makes $C X$ reach asymptote faster than $X$ does, but, once $X$ has also reached asymptote, both values are unaffected by the ratio between cause-present and cause-absent trials as long as $P(E \mid C)$ and $P(E \mid \sim C)$ are held constant (the same occurs with RW). Figure 3 displays a trial-by-trial simulation of Pearce's model for two conditions with equal $P(E \mid C)$ and $P(E \mid \sim C)$. In Condition $16 / 96$, the cause was present in only 16 of the 96 trials of the series (trial type frequencies were $\boldsymbol{a}=16, \boldsymbol{b}=0$, $\boldsymbol{c}=40, \boldsymbol{d}=40$ ), whereas in Condition 80/96, the cause was present in 80 of the 96 trials of the series $(\boldsymbol{a}=80$, $\boldsymbol{b}=0, \boldsymbol{c}=8, \boldsymbol{d}=8$ ). The parameters selected for these simulations were $x_{1}=.7, x_{2}=.5, x_{3}=.7, \beta=.25$, and $\lambda=1$ (see Equations 7-9). As can be readily noticed, the predicted associative strength increases much faster in Condition 80/96, but after 75 trials, the two curves are virtually indistinguishable. Exactly the same prediction derives from RW.

In contrast, the EI rule predicts a monotonic cause density effect in a number of situations. In Condition 16/96, for instance, there are 56 confirmatory instances $(\boldsymbol{a}=16$, $\boldsymbol{d}=40)$, and the proportion of confirmatory evidence is 56:96, whereas in Condition 80/96, there are 88 confirmatory instances $(\boldsymbol{a}=80, \boldsymbol{d}=8)$ and a ratio of 88:96 for confirmatory evidence (with regard to a generative hypothesis). As we will detail below, in this model, trial type weighting can be a different source of cause density effects, and the two can even modulate each other.

In relation to the cause density effect, the structurallearning model deserves some extra consideration. At variance with the power PC, $\Delta P, \mathrm{HPN}$, and Pearce's (1987) models, this model predicts a significant effect of cause density. The intuitive reason for this is rather simple: In order to make a reliable decision on the basis of the log ratio underlying support, it is necessary for the participant to collect enough information about both $P(E \mid C)$ and $P(E \mid \sim C)$. In other words, the sample of observations on which support is computed must be large, but, at the same time, the ratio between $\sim C$ and $C$ must not favor one trial type over another (at least not to a very high degree); a 


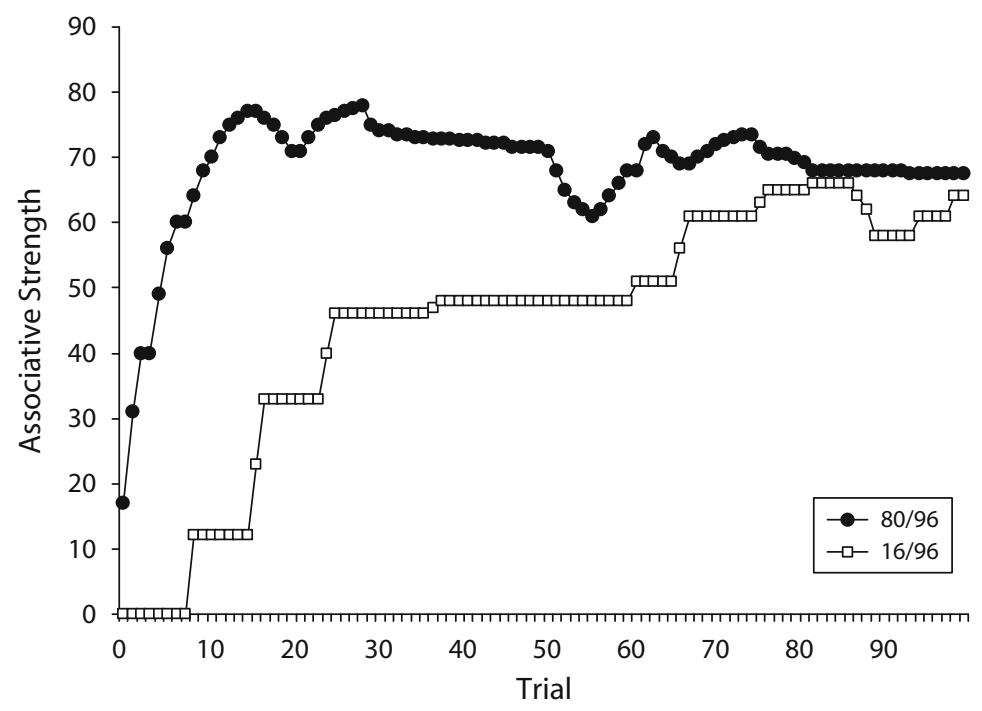

Figure 3. Pearce's (1987) model. Predicted associative strength across 96 trials in two conditions with low (16/96) and high (80/96) cause density.

significant reduction in both the number of $\sim C$ trials and (particularly) in the number of $C$ trials will reduce support. Cause density is expected not only to influence judgments linearly but also quadratically (yielding a slightly skewed bell shape), and this effect is expected to be stronger as the global sample size grows. As can be seen in the Appendix, in those cases in which the cause density effect shows up, the predicted bell shape does not appear, and judgments tend to increase with cause density (although perhaps not linearly).

Effects of trial type frequencies. The trends mentioned above (the cause density effect, the outcome density effect, and the sufficiency bias) result from orthogonal manipulations of each of the variables, but these trends in fact seem to interact. For example, in a recent study by Perales et al. (2005), the cause density effect was shown to depend on the degree of contingency between the cause and the effect, and it disappeared in null-contingency conditions. As noted by White (2004), these interactions could be due to the fact that none of the variables stand for the basic pieces of information on which causal induction processes are based (see also White, 2003c, and Mandel \& Lehman, 1998, for detailed descriptions of the origin of the cause density effect and its possible variants). The predictive advantage of the EI rule stems from the possibility of processing and weighting each trial type in the contingency table separately $\left(w_{a}>w_{b}>w_{c}>w_{d}\right.$; see Anderson \& Sheu, 1995; Levin, Wasserman, \& Kao, 1993) and on the way such pieces of information are combined. It can be shown that the sufficiency bias, the outcome density effect, and the orthogonal effects of $\Delta P$ and pow can be derived from the assumption that the four trial types are attributed different evidential weights. The cause density effect, in its several variants, seems to be a natural consequence of combining weighted trial type frequencies additively. If this is true, a complete characterization of judgments would require manipulating the frequency of each trial type independently and orthogonally (White, 2003a).

In order to test this idea, we carried out a regression analysis in which the frequencies of the four trial types across the 114 conditions in the meta-analysis were used as predictive variables, and the observed judgments were used as the dependent variables. Beta coefficients for $\boldsymbol{a}$, $\boldsymbol{b}, \boldsymbol{c}$, and $\boldsymbol{d}$ can be interpreted as estimates of the weights of the four frequencies on judgments. The resulting beta values are shown in Table 1 and reveal that $\boldsymbol{a}$ and $\boldsymbol{d}$ type events correlated positively with judgments, that $\boldsymbol{b}$ and $c$ type events correlated negatively with judgments, and that the ordering of absolute weights conformed to the customarily observed inequality $w_{a}>w_{b}>w_{c}>w_{d}$ (see also Perales et al., 2005).

The observed weighting pattern was compared with the one predicted by each of the models ( $p o w$, HPN, support, Pearce, RW, and EI). The parameters used to implement the models and compute the predicted judgments were those resulting from averaging the best-fitting parameters across the 1,000 iterations carried out in the (previously described) cross-validation procedure. A regression analysis of predicted judgments over the four trial type frequencies was carried out for each of the models; the absolute values of the standardized beta coefficients for each trial type frequency can be interpreted as ordinal indices of the predicted weights. In order to take support into account for this analysis, a power transformation (of the form $\left[j=\operatorname{sign}(\text { support)abs(support })^{k}\right]$ ) was used, where $k$ was searched in intervals of .05 to maximize the correlation between support and judgments $(k=.30 ; r=.81$; $\left.R^{2}=.66\right)$. This transformation is recommended by Griffiths and Tenenbaum (2005), since people's judgments tend to become asymptotic as the log likelihood ratio increases. Table 1 displays the weights for each model and allows them to be compared with the observed weights from participants' actual judgments. Only the EI rule 
matches the ordering of weightings usually found with standard causal judgments $\left(w_{a}>w_{b}>w_{c}>w_{d}\right)$. The power PC, HPN, Pearce, and RW models simply assign more weight to $\boldsymbol{a}$ and $\boldsymbol{b}$ type trials than to $\boldsymbol{c}$ and $\boldsymbol{d}$ type trials $\left(w_{a}=w_{b}>w_{c}=w_{d}\right)$, and the support model predicts a difference between $\boldsymbol{a}$ and the other frequencies but not a difference between $\boldsymbol{c}$ and $\boldsymbol{d}\left(w_{a}>w_{b}>w_{c}=w_{d}\right)$.

As mentioned above, it is possible that people's judgments reflect a conflation of parameter estimation and structure induction which could mean that some people use power (or its algorithmic version, HPN), and some use support to make their judgments. Since power is necessary for the computation of support, it could even be the case that people incorporate a version of power computed from biased probabilities into support when making their judgments. For strictly mathematical reasons, all of the variants of these possibilities would yield a weighting pattern in accordance with some combination of the weights predicted by pow, HPN, and support, none of which can fit the actual weighting pattern (especially the fact that $\left.w_{c}>w_{d}\right)$.

\section{DISCUSSION}

In the last few years, normative models of causal induction have become highly integrated and structured, thanks in part to the development of Bayesian graphical models in computer science and psychology. The power PC theory has emerged as the dominant computational theory for causal parameterization, whereas the structure-learning model is quickly becoming dominant for causal structure induction. The two models are highly compatible and make complementary predictions.

The evidence presented here, however, is bad news for this state of generalized agreement. The support model has substantial explanatory power when applied to standard causal judgments, but it fails to provide a complete account for the cause density effect and the customary weighting pattern $w_{a}>w_{b}>w_{c}>w_{d}$. These effects have been replicated several times in the past. Judgments seem to be directly influenced by cause density (given a specific value of objective contingency), and they do not vanish as the sample of observations grows (for a second, independent replication, see Perales et al., 2005), which contradicts the predictions of the structure model (support)

Table 1

Beta Weights, With Standard Deviations, Obtained From Regressing Observed and Predicted Judgments Onto the Event Frequencies $\boldsymbol{a}-\boldsymbol{d}$

\begin{tabular}{|c|c|c|c|c|c|c|c|c|}
\hline & \multicolumn{2}{|l|}{$a$} & \multicolumn{2}{|l|}{$b$} & \multicolumn{2}{|l|}{$c$} & \multicolumn{2}{|l|}{$d$} \\
\hline & Weight & $S D$ & Weight & $S D$ & Weight & $S D$ & Weight & $S D$ \\
\hline Observed & 0.60 & 0.12 & -0.40 & 0.09 & -0.30 & 0.12 & 0.15 & 0.09 \\
\hline pow & 0.48 & 0.12 & -0.52 & 0.09 & -0.21 & 0.09 & 0.25 & 0.12 \\
\hline $\mathrm{HPN}$ & 0.49 & 0.11 & -0.52 & 0.08 & -0.26 & 0.08 & 0.26 & 0.11 \\
\hline Pearce & 0.49 & 0.11 & -0.47 & 0.08 & -0.35 & 0.08 & 0.34 & 0.11 \\
\hline RW & 0.49 & 0.11 & -0.45 & 0.08 & -0.29 & 0.08 & 0.28 & 0.12 \\
\hline EI & 0.63 & 0.10 & -0.45 & 0.08 & -0.33 & 0.08 & 0.14 & 0.11 \\
\hline Support & 0.62 & 0.12 & -0.52 & 0.10 & -0.46 & 0.10 & 0.47 & 0.13 \\
\hline
\end{tabular}

Note-pow, causal power; HPN, hybrid pseudonormative model; RW, Rescorla-Wagner model; EI, evidence integration rule. and power PC (power). In consequence, the only method at hand that can account for standard judgments seems to be some sort of weighted linear combination of the four trial type frequencies in the contingency table, such as the EI rule.

The quantitative and qualitative model-fitting exercise presented here is also consistent in refuting almost all of the algorithmic models under consideration. Pearce's (1987) model (the most promising associative algorithm), along with the weighted version of $\Delta P$, yielded worse quantitative predictors of standard judgments than did the EI rule, and those two models cannot account for a number of consistent trends in the data, including the cause density effect and the pattern of weightings normally found in causal learning tasks. The HPN model, formulated here from Buehner et al.'s (2003) suggestion that causal judgments were influenced by both power and contingency, did well at quantitatively fitting the data from previous experiments but less well at predicting the observed rank ordering of judgments in each experiment. Most importantly, the HPN model failed again to predict the specific frequency weighting pattern consistently found across experiments.

If we accept that EI is the most predictive of the models under consideration, how does it relate to the computational goals of causal induction? As noted earlier, the EI rule and the log ratio underlying support model possess some mathematical similarities. Their qualitative predictions are affected by similar factors, and they assume similar trial type weightings, except that support overweights $\boldsymbol{d}$ type events. In addition, in both cases, a comparison is made between information confirming the hypothesis that a causal link exists and information indicating that that causal link does not exist. However, the similarity seems to go beyond this.

Prior to formulation of the structure-learning model, previous attempts to formulate a Bayesian analysis of simple causal induction (Anderson, 1990; Anderson \& Sheu, 1995) were made. Anderson's (1990) model is based on a Bayesian decision rule, but, interestingly, it allows us to establish a rational relationship between causal learning rules based on the linear combination of trial type frequencies (including the EI rule) and the goal of inferring structure.

In short, the main difference between support and Anderson's (1990) rational approach is how the causal structure is parameterized. The structure-learning model requires two parameters, $w_{1}$ and $w_{0}$, to represent the causal powers of the candidate cause $C$ and the set of alternative causes $A$ as well as the assumption that those powers combine noninteractively. In Anderson's model (Anderson \& Sheu, 1995), the reasoner is assumed to assign a certain a priori value to the probability of the effect in the presence of the cause $\left[P^{\prime}(E \mid C)\right]$ (note that this is a subjective belief rather than an objective probability, hence the change of notation) and another value to the probability of the effect in the absence of the cause $\left[P^{\prime}(E \mid \sim C)\right]$, under the a priori assumption that a causal link between the candidate cause and the effect $(H)$ exists. At the same time, the reasoner assigns a value to the base rate of the effect 
$\left[P^{\prime}(E)\right]$, which is not dependent on the presence or absence of the cause, under the assumption that the causal link between the candidate cause and the effect does not exist $(\sim H)$. Next, a decision about whether the data favor $H$ or $\sim H$ must be made (which amounts to the same thing as deciding between $\mathrm{Graph}_{1}$ and $\mathrm{Graph}_{0}$ in Griffiths \& Tenenbaum's 2005 notation).

At variance with the structure-learning model, this model includes three free parameters, representing the fixed priors $P^{\prime}(E \mid C), P^{\prime}(E \mid \sim C)$, and $P^{\prime}(E)$, but has some advantages. First, Anderson's (1990) model is not tied by default to the noisy-or schema; in principle, any model of cause-context interaction can be accommodated by the model. Second, this model provides a logical and mathematical basis for the weighted linear combination of frequencies. The model is expressed in the following equation:

$$
\begin{aligned}
\log \frac{P(H \mid D)}{P(\sim H \mid D)}= \\
\quad \log \frac{P(H)}{P(\sim H)} \\
\quad+\boldsymbol{a}\left[\log \left(P^{\prime}(E \mid C)\right)-\log \left(P^{\prime}(E)\right)\right] \\
\quad+\boldsymbol{b}\left[\log \left(1-P^{\prime}(E \mid C)\right)-\log \left(1-P^{\prime}(E)\right)\right] \\
\quad+\boldsymbol{c}\left[\log \left(P^{\prime}(E \mid \sim C)\right)-\log \left(P^{\prime}(E)\right)\right] \\
\quad+\boldsymbol{d}\left[\log \left(1-P^{\prime}(E \mid \sim C)\right)-\log \left(1-P^{\prime}(E)\right)\right] .
\end{aligned}
$$

From this equation alone, it is immediately apparent that the log odds on which the structural decision is made result from linearly combining the values of the four cells of the contingency table. The factors multiplying the trial type frequencies are equivalent to the factors multiplying each independent variable in a linear regression rule. At variance with the linear regression rule, however, the model specifies that if three of the four factors are known, the fourth is fixed, and therefore there are only three degrees of freedom in the model (instead of four).

As stated by Anderson and Sheu (1995, p. 513), "Equation [13] shows that rather sophisticated Bayesian inference can be achieved by a very simple response rule." The advantage of this rule is that, even if the noisy-or parameterization is refuted by experimental results, it can still relate the algorithm people use to the computational goal of making a structural decision on the existence or nonexistence of a hypothesized causal link. In consequence, a sensibly weighted linear combination of trial type frequencies (including EI, which uses normalization for scale adjustment) will provide an approximation of the output of a rational Bayesian strategy. In other words, EI and related rules (e.g., the $p C I$ rule or weighted $\Delta D$ ) stand as cognitively plausible heuristics to attain a rational computational goal-namely, to make an accurate structural judgment.

The similarity between Anderson's (1990) Bayesian rule and models from the EI family provide a normative justification for the way in which trial type frequencies are combined in such models. However, the ultimate (psychological) explanation for the fact that people consistently weight trials in the order $w_{a}>w_{b}>w_{c}>w_{d}$ remains open. In the Bayesian rule (Equation 13), trial type weights depend on the values assigned to $P^{\prime}(E \mid C), P^{\prime}(E \mid \sim C)$, and $P^{\prime}(E)$. If $H$ is supposed to imply a high probability of the effect when the cause is present (e.g., $P^{\prime}(E \mid C)=.7$ ) and a low probability of the effect when the cause is absent (e.g., $P^{\prime}(E \mid \sim C)=.2$ ), and $\sim H$ is supposed to imply a low base-rate of the effect (e.g., $P^{\prime}(E)=.3$ ), then the pattern of weights coincides with that customarily found in judgments. In other scenarios (for example, when the base rate of the effect is assumed to be high), the pattern can adopt a different shape. However, to our knowledge, there is no evidence that patterns of weighting vary as a function of base rate. In addition, judgments seem to be less sensitive to sample size than predicted by the Bayesian rule. These two facts would imply that people are not applying the Bayesian rule sensu stricto, but some heuristic rule that approximates it closely. An interesting question for further investigation is to ascertain whether that rule is attained by means of progressive interaction with the world and is thus dependent on learning factors.

\section{CONCLUSION}

The present article aimed to provide a demanding test of models in the field of simple covariation-based causal induction. Graphical models allow us to precisely describe the computational goals of causal induction and to permit the integration of power PC and the structure-learning model. However, some of the core assumptions of these two models have been strongly refuted, and, instead, a combinatory heuristic such as the EI rule appears to be more predictive of actual judgments than any other normbased or algorithmic model.

We are aware that a number of important questions are left unanswered. First, our analyses are restricted to onecause, one-effect trial-by-trial tasks, which could limit the generality of the observed results. However, exhaustive analyses of the mapping between trial type frequencies and causal judgments in paradigms in which the covariation information is provided simultaneously (instead of sequentially) yield results essentially identical to ours (White, 2003c, 2004). At the same time, a rule for multiple-cause scenarios based on the $p C I$ model (which is largely similar to EI) has recently been proposed (White, 2005a, 2005b). Second, we put off the question of whether counterfactual judgments can be regarded as a useful tool for investigating parameter induction. And third, we do not make any strong assumptions about the ultimate psychological explanation of weighting patterns.

In consequence, our interpretation is that the EI rule is justified on rational and empirical grounds and provides clear recommendations for future research - namely, more attention should be paid to task requirements in relation to clearly specified computational goals, and results should be integrated into a coherent computational framework.

\section{AUTHOR NOTE}

This research was supported by the Spanish Ministry of Education's program Becas Postdoctorales en España y el Extranjero: Convocatoria 2003, Ministerio de Ciencia y Tecnología Grant BSO2003-03723, and Ministerio de Educación y Ciencia Grant SEJ2006-11906/PSIC) to 
the first author, and by a grant from the United Kingdom Economic and Social Research Council to the second author. We thank David Lagnado and Mark Johansen for their many helpful comments. We also thank Andrés Catena for his help in conducting the simulations for the structure-learning model, Tom Griffiths for his valuable comments on the interpretation of that model, and Leyre Castro for comments on and assistance with the work described here. Correspondence regarding this article should be addressed to J. C. Perales, Facultad de Psicología, Campus de Cartuja, s/n 18071, Granada, Spain (e-mail: jcesar@ugr.es).

\section{REFERENCES}

Aitken, M. R. F., Larkin, M. J. W., \& Dickinson, A. (2000). Superlearning of causal judgements. Quarterly Journal of Experimental Psychology, 53B, 59-81.

Aitken, M. R. F., Larkin, M. J. W., \& Dickinson, A. (2001). Reexamination of the role of within-compound associations in the retrospective revaluation of causal judgements. Quarterly Journal of Experimental Psychology, 54B, 27-51.

ALLAN, L. G. (1993). Human contingency judgments: Rule based or associative? Psychological Bulletin, 114, 435-448.

Allan, L. G. (2003). Assessing power PC. Learning \& Behavior, 31, 192-204.

Allan, L. G., \& Jenkins, H. M. (1983). The effect of representations of binary variables on judgment of influence. Learning \& Motivation, 14, 381-405.

ANDERSON, J. R. (1990). The adaptive character of thought. Hillsdale, NJ: Erlbaum.

Anderson, J. R., \& Sheu, C.-F, (1995). Causal inferences as perceptual judgments. Memory \& Cognition, 23, 510-524.

Baker, A. G., VAllée-Tourangeau, F., \& MurPhy, R. A. (2000). Asymptotic judgment of cause in a relative validity paradigm. Memory \& Cognition, 28, 466-479.

Buehner, M. J., \& CHENG, P. W. (1997). Causal induction: The power PC theory versus the RW model. In M. G. Shafto \& P. Langley (Eds.), Proceedings of the Nineteenth Annual Conference of the Cognitive Science Society (pp. 55-69). Hillsdale, NJ: Erlbaum.

Buehner, M. J., Cheng, P. W., \& Clifford, D. (2003). From covariation to causation: A test of the assumption of causal power. Journal of Experimental Psychology: Learning, Memory, \& Cognition, 29, 1119-1140.

BUEHNER, M. J., \& MAY, J. (2003). Rethinking temporal contiguity and the judgement of causality: Effects of prior knowledge, experience, and reinforcement procedure. Quarterly Journal of Experimental Psychology, 56A, 865-890.

BUSEMEYER, J. R. (1991). Intuitive statistical estimation. In N. H. Anderson (Ed.), Contributions to information integration theory (pp. 187205). Hillsdale, NJ: Erlbaum.

Catena, A., Maldonado, A., \& Cándido, A. (1998). The effect of frequency of judgement and the type of trials on covariation learning. Journal of Experimental Psychology: Human Perception \& Performance, 24, 481-495.

Chapman, G. B., \& Robins, S. J. (1990). Cue interaction in human contingency judgment. Memory \& Cognition, 18, 537-545.

Cheng, P. W. (1993). Separating causal laws from causal facts: Pressing the limits of statistical relevance. In D. L. Medin (Ed.), The psychology of learning and motivation: Advances in research and theory (Vol. 30, pp. 215-264). San Diego: Academic Press.

Cheng, P. W. (1997). From covariation to causation: A causal power theory. Psychological Review, 104, 367-405.

Cheng, P. W. (2000). Causality in the mind: Estimating contextual and conjunctive power. In F. C. Keil \& R. A. Wilson (Eds.), Explanation and cognition (pp. 227-253). Cambridge, MA: MIT Press.

Cheng, P. W., \& Holyonk, K. J. (1995). Complex adaptive systems as intuitive statisticians: Causality, contingency, and prediction. In H. L. Roitblat \& J.-A. Meyer (Eds.), Comparative approaches to cognitive science (pp. 271-302). Cambridge, MA: MIT Press.

Cheng, P. W., \& Novick, L. R. (1992). Covariation in natural causal induction. Psychological Review, 99, 365-382.

Collins, D. J., \& Shanks, D. R. (2006). Conformity to the power PC theory of causal induction depends on type of probe question. Quarterly Journal of Experimental Psychology, 59, 225-232.
DANKs, D. J. (2002). The epistemology of causal judgment. Dissertation Abstracts International, 63, 212.

De Houwer, J., \& Beckers, T. (2002). A review of recent developments in research and theories on human contingency learning. Quarterly Journal of Experimental Psychology, 55B, 289-310.

DiCKINSON, A., \& BURKE, J. (1996). Within-compound associations mediate the retrospective revaluation of causality judgements. Quarterly Journal of Experimental Psychology, 49B, 60-80.

GLAUTIER, S. (2002). Spatial separation of target and competitor cues enhances blocking of human causality judgements. Quarterly Journal of Experimental Psychology, 55B, 121-135.

GLYMOUR, C. (1998). Learning causes: Psychological explanations of causal explanation. Mind \& Machines, 8, 39-60.

Glymour, C. (2001). The mind's arrows: Bayes nets and graphical causal models in psychology. Cambridge, MA: MIT Press.

Glymour, C., Scheines, R., Spirtes, P., \& Kelly, K. (1987). Discovering causal structure: Artificial intelligence, philosophy of science, and statistical modeling. San Diego: Academic Press.

Griffiths, T. L., \& Tenenbaum, J. B. (2005). Structure and strength in causal induction. Cognitive Psychology, 51, 334-384.

INHELDER, B., \& PIAGET, J. (1958). The growth of logical thinking from childhood to adolescence: An essay on the construction of formal operational structures. Oxford: Basic Books.

Lagnado, D., \& Sloman, S. A. (2004). The advantage of timely intervention. Journal of Experimental Psychology: Learning, Memory, \& Cognition, 30, 856-876.

LEVIN, I. P., WassERMan, E. A., \& KaO, S.-F. (1993). Multiple methods for examining biased information use in contingency judgments. $\mathrm{Or}$ ganizational Behavior \& Human Decision Processes, 55, 228-250.

LoBER, K., \& ShanKs, D. R. (2000). Is causal induction based on causal power? Critique of Cheng (1997). Psychological Review, 107, 195-212.

LOVIBOND, P. F. (2003). Causal beliefs and conditioned responses: Retrospective revaluation induced by experience and by instruction. Journal of Experimental Psychology: Learning, Memory, \& Cognition, 29,97-106.

Maldonado, A., Jiménez, G., Herrera, A., Perales, J. C., \& CaTENA, A. (2006). Inattentional blindness for negative relationships in human causal learning. Quarterly Journal of Experimental Psychology, 59, 457-470.

MANDEL, D. R., \& LeHMAN, D. R. (1998). Integration of contingency information in judgments of cause, covariation, and probability. Journal of Experimental Psychology: General, 127, 269-285.

MYUnG, I. J., Foster, M. R., \& Browne, M. W. (EDS.) (2000). Special issue on model selection. Journal of Mathematical Psychology, 44, $1-231$.

PEARCE, J. M. (1987). A model for stimulus generalization in Pavlovian conditioning. Psychological Review, 94, 61-73.

Pearl, J. (1988). Probabilistic reasoning in intelligent systems. San Francisco: Morgan Kaufmann.

Pearl, J. (2000). Causality: Models, reasoning, and inference. New York: Cambridge University Press.

Perales, J. C., \& Catena, A. (2006). Human causal induction: A glimpse at the whole picture. European Journal of Cognitive Psychology, 18, 277-320.

Perales, J. C., Catena, A., \& Maldonado, A. (2004). Inferring non-observed correlations from causal scenarios: The role of causal knowledge. Learning \& Motivation, 35, 115-135.

Perales, J. C., Catena, A., Shanks, D. R., \& González, J. A. (2005). Dissociation between judgments and outcome-expectancy measures in covariation learning: A signal detection theory approach. Journal of Experimental Psychology: Learning, Memory, \& Cognition, 31, 1105-1120.

Perales, J. C., \& Shanks, D. R. (2003). Normative and descriptive accounts of the influence of power and contingency on causal judgement. Quarterly Journal of Experimental Psychology, 56A, 977-1007.

PeRALES, J. C., \& ShanKs, D. R. (2004, May). The cause-density effect as a tool to discriminate between causal learning models. Paper presented at the Special Interest Meeting on Human Contingency Learning, Lignely, Belgium.

Rescorla, R. A., \& Wagner, A. R. (1972). A theory of Pavlovian conditioning: Variations of the effectiveness of reinforcement and nonreinforcement. In A. H. Black \& W. F. Prokasy (Eds.), Classical 
conditioning II: Current research and theory (pp. 64-99). New York: Appleton-Century-Crofts.

Schustack, M. W., \& Sternberg, R. J. (1981). Evaluation of evidence in causal inference. Journal of Experimental Psychology: General, 110, 101-120.

SHANKS, D. R. (1995). The psychology of associative learning. New York: Cambridge University Press.

Shanks, D. R. (2002). Tests of the power PC theory of causal induction with negative contingencies. Experimental Psychology, 49, 81-88.

Shanks, D. R., \& Dickinson, A. (1987). Associative accounts of causality judgment. In G. H. Bower (Ed.), The psychology of learning and motivation: Vol. 21. Advances in research and theory (pp. 229-261). San Diego: Academic Press.

Shanks, D. R., Holyoak, K., \& Medin, D. L. (Eds.) (1996). The psychology of learning and motivation: Vol. 34. Causal learning. San Diego: Academic Press.

Shanks, D. R., Pearson, S. M., \& Dickinson, A. (1989). Temporal contiguity and the judgement of causality by human subjects. Quarterly Journal of Experimental Psychology, 41B, 139-159.

Sloman, S. A. (2005). Causal models: How people think about the world and its alternatives. Oxford: Oxford University Press.

Sloman, S. A., \& LaGnado, D. (2004). Causal invariance in reasoning and learning. In B. Ross (Ed.), The psychology of learning and motivation: Vol. 44. Advances in research and theory (pp. 207-325). San Diego: Academic Press.

Smedslund, J. (1963). The concept of correlation in adults. Scandinavian Journal of Psychology, 4, 165-173.

SPIRTES, P., GlymOUR, C., \& ScheINes, R. (1998). Causation, prediction, and search. Cambridge, MA: MIT Press.

Steyvers, M., Tenenbaum, J. B., Wagenmakers, E.-J., \& Blum, B. (2003). Inferring causal networks from observations and interventions. Cognitive Science, 27, 453-489.

Tenenbaum, J. B., \& GRIFFiths, T. L. (2001). Structure learning in human causal induction. In T. K. Leen, T. G. Dietterich, \& V. Tresp (Eds.), Advances in neural information processing systems (Vol. 13, pp. 59-65). Cambridge, MA: MIT Press.

Vallée-Tourangeau, F., Murphy, R. A., Drew, S., \& Baker, A. G. (1998). Judging the importance of constant and variable candidate causes: A test of the power PC theory. Quarterly Journal of Experimental Psychology, 51A, 65-84.

VAN-HAMme, L. J., \& WASSERMan, E. A. (1994). Cue competition in causality judgments: The role of nonpresentation of compound stimulus elements. Learning \& Motivation, 25, 127-151.

WaLdMAnN, M. R. (2000). Competition among causes but not effects in predictive and diagnostic learning. Journal of Experimental Psychology: Learning, Memory, \& Cognition, 26, 53-76.

WALDMANN, M. R., \& HAGMAYER, Y. (2001). Estimating causal strength: The role of structural knowledge and processing effort. Cognition, 82, 27-58.

WALDMANN, M. R., \& HolyoAK, K. J. (1992). Predictive and diagnostic learning within causal models: Asymmetries in cue competition. Journal of Experimental Psychology: General, 121, 222-236.

WALDMANN, M. R., \& HolyOAK, K. J. (1997). Determining whether causal order affects cue selection in human contingency learning: Comments on Shanks and Lopez (1996). Memory \& Cognition, 25, 125-134.

WASSERMAN, E. A. (1993). Comparative cognition: Toward a general understanding of cognition in behavior. Psychological Science, $\mathbf{4}$, 156-161.

Wasserman, E. A., Kao, S.-F., Van-Hamme, L. J., Katagiri, M., \& YounG, M. E. (1996). Causation and association. In D. R. Shanks, K. J. Holyoak, \& D. L. Medin (Eds.), The psychology of learning and motivation: Vol. 34. Causal learning (pp. 207-264). San Diego: Academic Press.

White, P. A. (2003a). Causal judgement as evaluation of evidence: The use of confirmatory and disconfirmatory information. Quarterly Journal of Experimental Psychology, 56A, 491-513.
White, P. A. (2003b). Effects of wording and stimulus format on the use of contingency information in causal judgment. Memory \& Cognition, 31, 231-242.

WHITE, P. A. (2003c). Making causal judgments from the proportion of confirming instances: The pCI rule. Journal of Experimental Psychology: Learning, Memory, \& Cognition, 29, 710-727.

W HITE, P. A. (2004). Causal judgment from contingency information: A systematic test of the $p$ CI rule. Memory \& Cognition, 32, 353-368.

White, P. A. (2005a). Cue interaction effects in causal judgment: An interpretation in terms of the evidential evaluation model. Quarterly Journal of Experimental Psychology, 58B, 99-140.

WHITE, P. A. (2005b). Judgement of two causal candidates from contingency information: II. Effects of information about one cause on judgements of the other cause. Quarterly Journal of Experimental Psychology, 58A, 999-1021.

\section{NOTES}

1. Note the distinction between the causal events $A$ and $C$ and the cell frequencies $\boldsymbol{a}$ and $\boldsymbol{c}$.

2. In fact, if the parameters are removed from the denominator, a bizarre prediction arises: Even if a trial type is attributed a low weight (i.e., if it is considered relatively less informative for making a causal judgment), that trial type is still considered to define the number of instances over which the contrast is computed. Imagine a reasoner who considers $\boldsymbol{d}$ type trials as neither confirmatory nor disconfirmatory (i.e., $\boldsymbol{d}$ is attributed a zero weight) in a task in which $\boldsymbol{a}=10, \boldsymbol{b}=0, \boldsymbol{c}=2$, and $\boldsymbol{d}=25$. If the weights are not included in the denominator, the global judgment for that participant would be abnormally low-approximately $9 / 25$ with the usual weights - whereas if weights are included in the denominator, the judgment would be much higher-approximately 9/11. Although the versions do not differ in terms of qualitative predictiveness, the one used in the remainder of this article (equivalent to the information integration model; Busemeyer, 1991) allows better quantitative predictions.

3. The original formulation of Pearce's (1987) model does not consider the possibility of assigning different parameters to the degrees of generalization from $C X$ to $X$ and from $X$ to $C X$. This possibility is, however, compatible with the assumptions of the theory. According to Pearce (1987), generalization depends on the number of perceptual features two stimuli share: If $C X$ is activated, all of the elements of $X$ will also be activated, whereas if $X$ is activated, only some of the elements of $C X$ will be activated. This is due to the fact that $X$ is completely subsumed by $C X$, and therefore generalization can be considered to be asymmetrical. Leaving this possibility open is intentional and is aimed at testing the model at its most general (in any case, the effect of model complexity is reduced by cross validation and does not necessarily imply a competitive advantage).

4. A nonlinear transformation of the output of each model would add at least one parameter to each of them. Obviously, this addition would not alter the ordering of judgments predicted by any model for any set of conditions, although it might affect the quantitative fitting of judgments to data. In addition, without knowing the exact function that maps algorithm outputs onto judgments, applying one type of transformation or another would be arbitrary and void of theoretical content. In any case, we determined that a power transformation does not alter the ordering of models reported in the previous section. Thus, for the sake of simplicity, we kept the original formulations of all models. The implications of applying a power transformation to support are discussed below.

5. This effect is illustrated by the judgmental differences between Conditions 1 and 2 and between Conditions 3 and 4 in Experiment 1 from Perales and Shanks's (2003) study; among Conditions 1-3 in Experiment 3 from Lober and Shanks (2000); between Conditions 2 and 3 in Experiments 1 and 2 from Collins and Shanks (2006); among Conditions 2-4 from Experiment 3 in Shanks (2002); among Conditions 1-3 in Experiment 1 and Conditions 1-4 in Experiment 2 from Vallée-Tourangeau et al. (1998); between Conditions 1 and 4 and 2 and 3 in Experiment 6 from White (2003c); and between Conditions 2 and 3 and among Conditions 4-6, 7-10, and 11-13 in Experiment 1 from Buehner et al. (2003). 


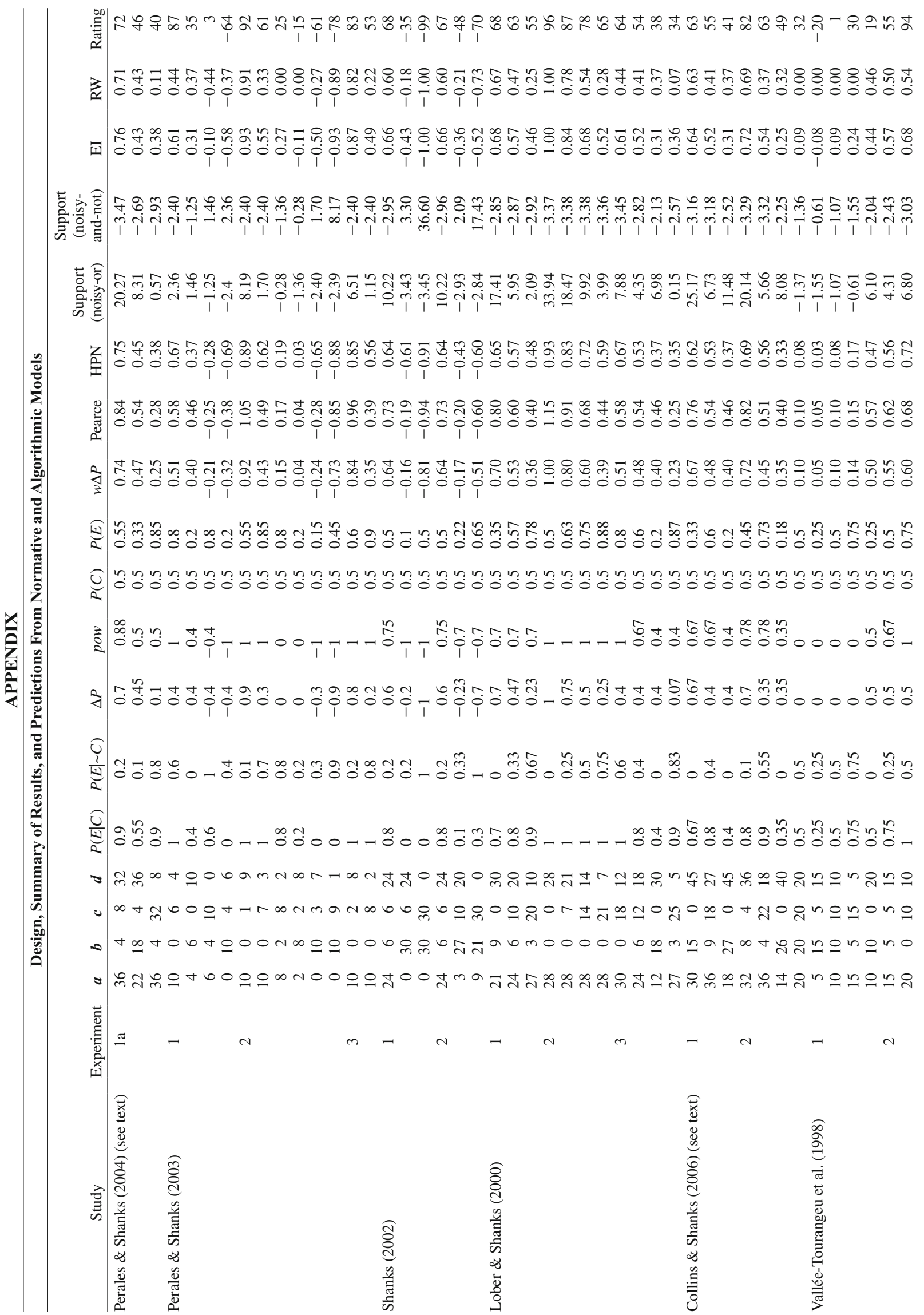




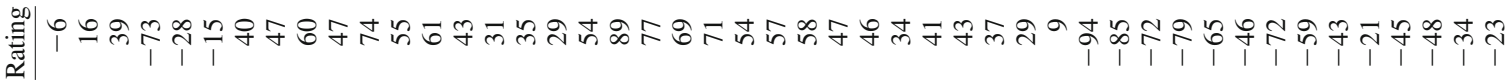

888 은

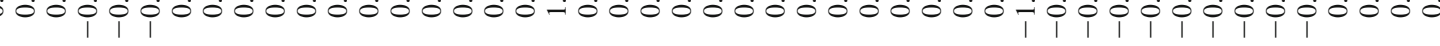

피

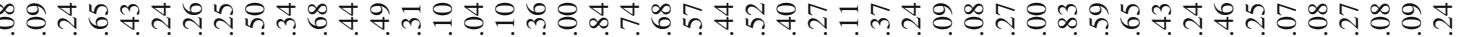
io.

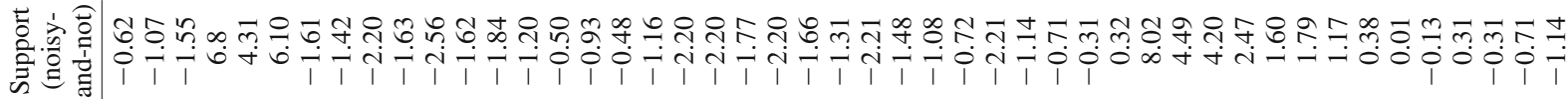

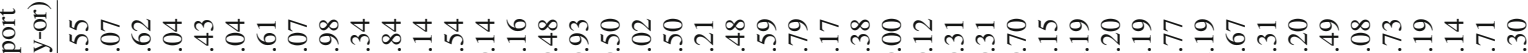
鵕け

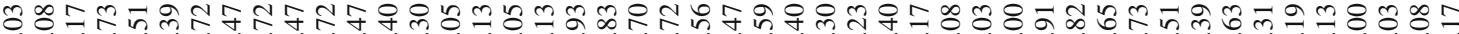

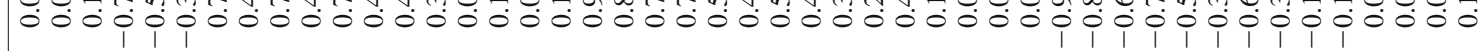

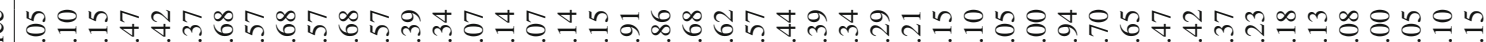

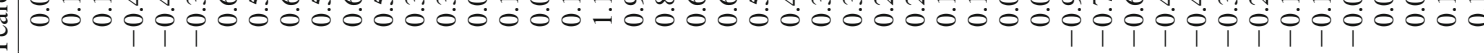

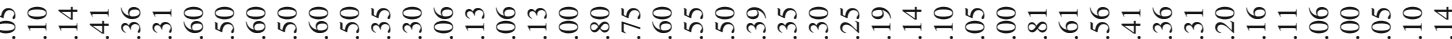

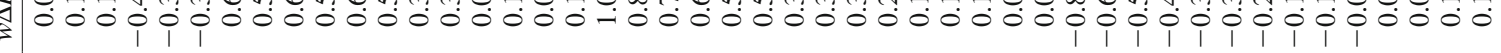

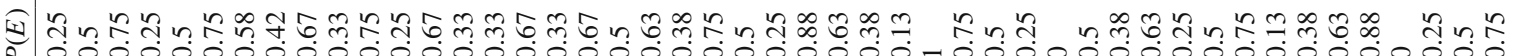
2 0000000000000000000000000000-000000000000000000

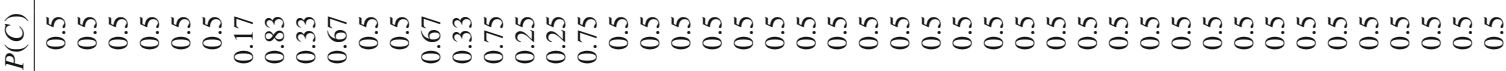

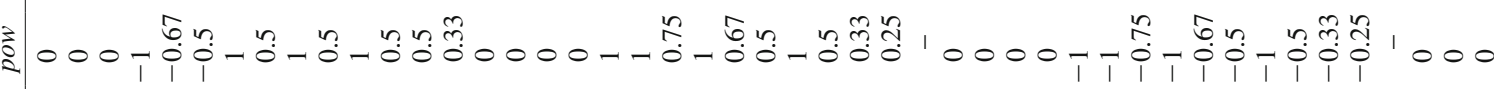
Ұ

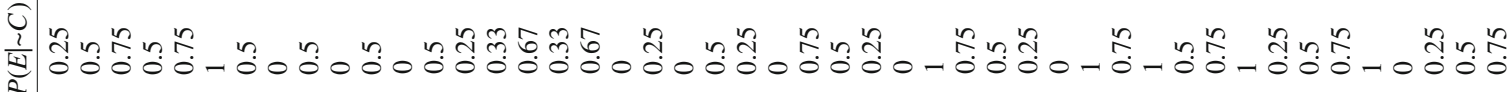

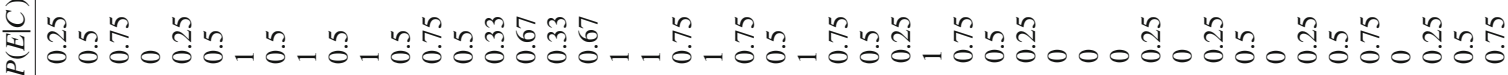

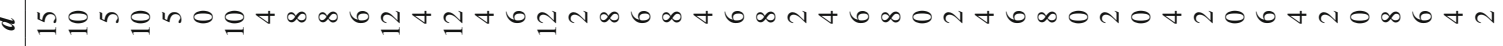

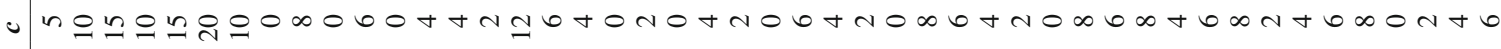

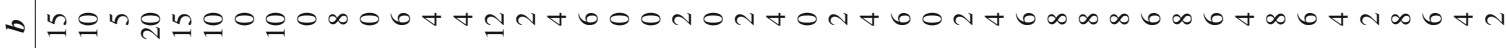

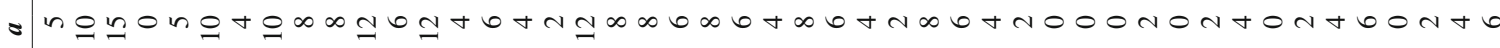



>ব 